\title{
Dynamics of Entanglement between a Quantum Dot Spin Qubit and a Photon Qubit inside a Semiconductor High-Q Nanocavity
}

\author{
Hubert Pascal Seigneur, ${ }^{1}$ Gabriel Gonzalez, ${ }^{2,3}$ \\ Michael Niklaus Leuenberger, ${ }^{2,3}$ and \\ Winston Vaughan Schoenfeld ${ }^{1}$ \\ ${ }^{1}$ CREOL College of Optics and Photonics, University of Central Florida, Orlando, FL 32826, USA \\ ${ }^{2}$ NanoScience Technology Center, University of Central Florida, Orlando, FL 32826, USA \\ ${ }^{3}$ Department of Physics, University of Central Florida, P. O. Box 162385, Orlando, FL 32816, USA
}

Correspondence should be addressed to Hubert Pascal Seigneur, seigneur@creol.ucf.edu

Received 15 September 2009; Accepted 24 November 2009

Academic Editor: Shao-Ming Fei

Copyright (C) 2010 Hubert Pascal Seigneur et al. This is an open access article distributed under the Creative Commons Attribution License, which permits unrestricted use, distribution, and reproduction in any medium, provided the original work is properly cited.

We investigate in this paper the dynamics of entanglement between a QD spin qubit and a single photon qubit inside a quantum network node, as well as its robustness against various decoherence processes. First, the entanglement dynamics is considered without decoherence. In the small detuning regime $(\Delta=78 \mu \mathrm{eV})$, there are three different conditions for maximum entanglement, which occur after 71, 93, and 116 picoseconds of interaction time. In the large detuning regime $(\Delta=1.5 \mathrm{meV})$, there is only one peak for maximum entanglement occurring at 625 picoseconds. Second, the entanglement dynamics is considered with decoherence by including the effects of spin-nucleus and hole-nucleus hyperfine interactions. In the small detuning regime, a decent amount of entanglement (35\% entanglement) can only be obtained within 200 picoseconds of interaction. Afterward, all entanglement is lost. In the large detuning regime, a smaller amount of entanglement is realized, namely, 25\%. And, it lasts only within the first 300 picoseconds.

\section{Introduction}

In order to continue satisfying Moore's law and sustain technological growth, a quantum approach that takes advantage of the wave nature of particles needs to be considered. Currently, the main problem is no longer the physical realization of the qubit, but rather the engineering of a practical quantum computing architecture or quantum network. As a consequence of decisive factors such as on-chip implementation, efficient transfer of quantum information, scalability, CMOS compatibility, and cost, the general consensus is that various implementations of the qubit should be combined in order to obtain an efficient quantum 
technology. This calls for qubits that are good for storage such as atoms to be used at quantum networks nodes while qubits that have desirable properties for travel such as photons as well as a coherence quantum interface between these qubits allowing for the exchange of information .

To the end of realizing an efficient quantum computing architecture, this promising composite qubit approach to a quantum technology has been proposed for ion trap [1] and also for neutral atoms [2]. We on the other hand have proposed a similar approach in connection with semiconductor-based artificial atoms or quantum dots (QDs) [3]. What does our scheme consist of? It consists of engineering a photonic crystal chip hosting a quantum network made of QDs spin embedded in defect cavities (storage qubits), which constitute the nodes of the network. These storage qubits can interact with other storage qubits at other locations or nodes by means of single photons (traveling qubits), which are guided through waveguides. Interestingly, this coherent interface, which is responsible for the state of the storage qubits to be mapped onto the traveling qubits or the entanglement between them, is itself a qubit system, the cavity-QED qubit (exchange or interaction qubit). Figure 1 depicts a storage qubit (an electron spin in a QD) interacting with a traveling qubit (a single photon) inside a quantum network node.

Our approach to a quantum network offers unique benefits with respect to the other composite qubit schemes. For instance, realistic on-chip implementation using photonic crystal has been shown to be plausible [4]; which is not the case in both the ion trap and neutral atom qubits. In addition, even though low temperatures are desirable for minimizing decoherence for the QD spin qubit, it is no where near the extreme temperatures needed for the functioning of the superconducting qubit. They are also much more robust against the influence of temperature than ion trap qubits. In fact, spin lifetimes up to 20 milliseconds have been reported [5]. This technology is easily scalable as additional nodes for the quantum network are generated by just creating additional cavities with embedded QDs in the photonic chip. Besides, because it is a semiconductor-based quantum technology, it is anticipated to be CMOS compatible and cost effective. The use of single photons as traveling qubits as well as the wavelengths considered makes not only the on-chip transfer of quantum information but also the long distance quantum communication by means of optical fibers efficient.

In these many regards, combining subwavelength photonic structures and semiconductor quantum dots provides an unmatched environment for the implementation of storage, exchange, and travelling qubits in comparison to other composite qubit schemes. Scaling up such existing technology by means of computer models is critical in order to build a fully functional quantum network. Such model is expected to lead to a thorough understanding of not only the coherent interaction between a QD and a single photon but also the entanglement dynamics between such particles produced inside quantum networks in a controlled way, in particular the fidelity or amount of such entanglement. For that reason, computer models are a fundamental step in gaining control over quantum information. As a result, we investigate in this paper the dynamics of entanglement between a QD spin qubit and a single-photon qubit inside a quantum network node, as well as its robustness against various decoherence processes.

\section{Theory of Quantum Network Nodes}

Modeling quantum network nodes requires the ability to describe the following physical systems (1) a QD, (2) a single-photon field, and (3) the interaction between these two in 


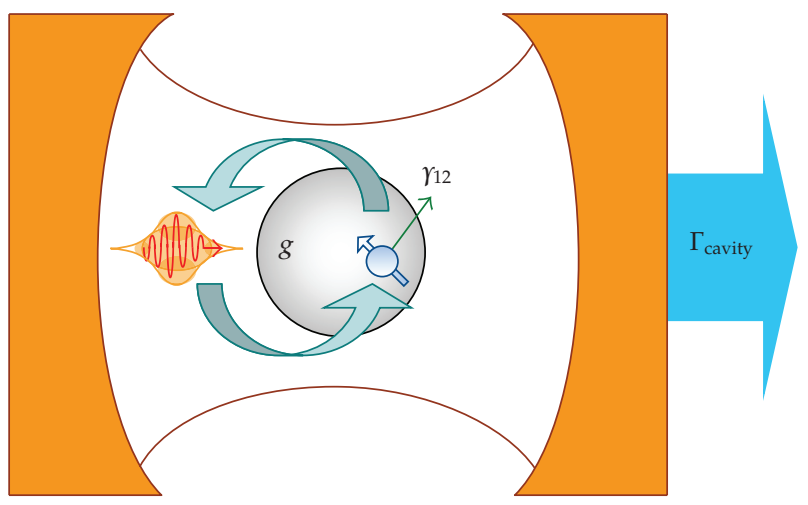

Figure 1: Storage, exchange and traveling qubits inside a quantum network node.

a nanocavity. How though are descriptions of the physical systems making up quantum network nodes used to implement the various qubits? First, the storage qubit is implemented using the spin states of a single excess electron in the conduction band of the QD. What about the traveling qubit? The polarization states of the single-photon in the single mode cavity, whether in the linear or circular polarization eigenbasis, could be used to describe the states of the traveling qubit. It turns out that the implementation of the quantum network nodes using semiconductor QD will necessitate the representation of the traveling qubit to be in the circular polarization eigenbasis. Last, the exchange qubit takes on the form of the "dressed" states in the "dressed" atom picture.

\subsection{Modified Jaynes-Cummings Model}

Quantum network nodes can be effectively described by a model very similar to the JaynesCummings Model. The main difference between the model for the quantum network nodes and the JC model is the construction of the two-level system, which it is 2-fold degenerate in the excited state and 4-fold degenerate in the ground state, shown in Figure 2. It is important to note that these four degenerate ground states are not connected specifically with light and heavy holes, although the selection rules are identical. We will refer to these degenerate states as $\left|h_{3 / 2}\right\rangle$ (2-fold degenerate valence band states with angular momentum projection $\pm 3 / 2$ ) and $\left|h_{1 / 2}\right\rangle$ (2-fold degenerate valence band states with angular momentum projection $\pm 1 / 2$ ) $[6,7]$. It is assumed that the QD is spherical in shape resulting in the confining potential with symmetry approximately identical to that of the first Brillouin zone of the anticipated cubic lattice of the semiconductor crystal. Accordingly, only a shift in the energy levels occurs while the degeneracy between $\left|h_{3 / 2}\right\rangle$ and $\left|h_{1 / 2}\right\rangle$ states is conserved (A degeneracy lift would not prevent this scheme from working [8], it would only change the time and condition necessary to perform entanglement.) When idle, the quantum dot system has all its ground state levels occupied while only one electron occupies the 2 -fold degenerate excited state resulting ideally in an "infinite" decay time. The ability for the excited state to retain this electron is critical to store and process quantum information in our scheme as we will see in the rest of this section. Last, unlike the Jaynes-Cummings Model, the model for the quantum network nodes will take account of various decoherence processes.

Because the QD system as a pseudo two-level system can be expressed in the total angular momentum (orbital angular momentum and spin) eigenbasis, there are clearly 


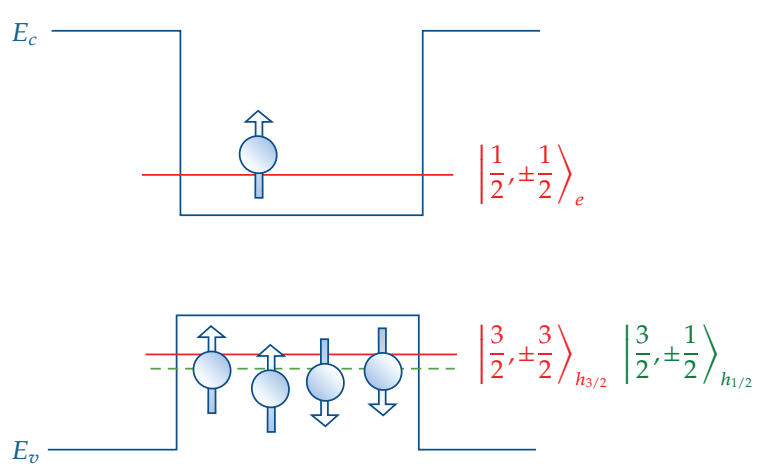

Figure 2: Two-level approximation of QD for the quantum network nodes.

defined optical transition rules for the electrical dipole interaction between ground and excited states, namely, that the orbital angular momentum quantum number $l$ changes by \pm 1 , and the spin is conserved as well as the parity of the envelop function. Furthermore, it follows that in the Faraday geometry, which requires the quantization axis of the excess electron spin to be parallel to the direction of light propagation, the empty state in the conduction band is only populated by circular polarized light (either $\left|\sigma^{+}\right\rangle$or $\left|\sigma^{-}\right\rangle$depending on the excess electron spin state). This is illustrated in Figure 3 for the case when the excess electron spin is initialized to $|\uparrow\rangle$.

Consequently, the total Hamiltonian for describing the quantum network node system is thus written as

$$
\widehat{\mathbf{H}}=\widehat{\mathbf{H}}_{\mathrm{QD}}+\widehat{\mathbf{H}}_{\text {Photon }}+\widehat{\mathbf{H}}_{\mathrm{QD}-\text { Photon }}
$$

where

$$
\begin{aligned}
& \widehat{\mathbf{H}}_{\mathrm{QD}}=\hbar \omega_{3 / 2} \widehat{\boldsymbol{\sigma}}_{3 / 2 \mathbf{v}, 3 / 2 \mathbf{v}}+\hbar \omega_{3 / 2} \widehat{\boldsymbol{\sigma}}_{-3 / 2 \mathbf{v},-3 / 2 \mathbf{v}} \\
& +\hbar \omega_{1 / 2} \widehat{\sigma}_{1 / 2 \mathrm{v}, 1 / 2 \mathrm{v}}+\hbar \omega_{1 / 2} \widehat{\sigma}_{-1 / 2 \mathrm{v},-1 / 2 \mathrm{v}} \\
& +\hbar \omega_{e} \widehat{\sigma}_{1 / 2 c, 1 / 2 c}+\hbar \omega_{e} \widehat{\sigma}_{-1 / 2 c,-1 / 2 c} \\
& \widehat{\mathbf{H}}_{\text {Photon }}=\hbar v \widehat{\mathbf{a}}_{\sigma+}^{\dagger} \widehat{\mathbf{a}}_{\sigma+}+\hbar v \widehat{\mathbf{a}}_{\sigma-}^{\dagger} \widehat{\mathbf{a}}_{\sigma-}, \\
& \widehat{\mathbf{H}}_{\mathrm{QD}-\text { Photon }}=\hbar g_{3 / 2} \widehat{\mathbf{a}}_{\boldsymbol{\sigma}+}^{\dagger} \widehat{\boldsymbol{\sigma}}_{-3 / 2 \mathbf{v},-1 / 2 \mathbf{c}}+\hbar g_{3 / 2} \widehat{\mathbf{a}}_{\boldsymbol{\sigma}-}^{\dagger} \widehat{\boldsymbol{\sigma}}_{3 / 2 \mathbf{v}, \mathbf{1} / 2 \mathbf{c}} \\
& +\hbar g_{3 / 2} \widehat{\boldsymbol{\sigma}}_{-1 / 2 \mathrm{c},-3 / 2 \mathrm{v}} \widehat{\mathbf{a}}_{\sigma+}+\hbar g_{3 / 2} \widehat{\boldsymbol{\sigma}}_{1 / 2 \mathrm{c}, 3 / 2 \mathrm{v}} \widehat{\mathbf{a}}_{\sigma-} \\
& +\hbar g_{1 / 2} \widehat{\mathbf{a}}_{\sigma+}^{\dagger} \widehat{\boldsymbol{\sigma}}_{-1 / 2 \mathrm{v}, 1 / 2 \mathrm{c}}+\hbar g_{1 / 2} \widehat{\mathbf{a}}_{\sigma-}^{\dagger} \widehat{\boldsymbol{\sigma}}_{1 / 2 \mathrm{v},-1 / 2 \mathrm{c}} \\
& +\hbar g_{1 / 2} \widehat{\sigma}_{1 / 2 \mathrm{c},-1 / 2 \mathrm{v}} \widehat{\mathbf{a}}_{\sigma+}+\hbar g_{1 / 2} \widehat{\sigma}_{-1 / 2 \mathrm{c}, 1 / 2 \mathrm{v}} \widehat{\mathbf{a}}_{\sigma-} .
\end{aligned}
$$

The various coupling strengths from valence band states with total angular momentum $j=$ $\pm 3 / 2$ to conduction band states with total angular momentum $j= \pm 1 / 2$ are be denoted $g_{3 / 2}$, and the various coupling strengths from valence band states with total angular momentum $j= \pm 1 / 2$ to conduction band states with total angular momentum $j= \pm 1 / 2$ are denoted $g_{1 / 2}$. 

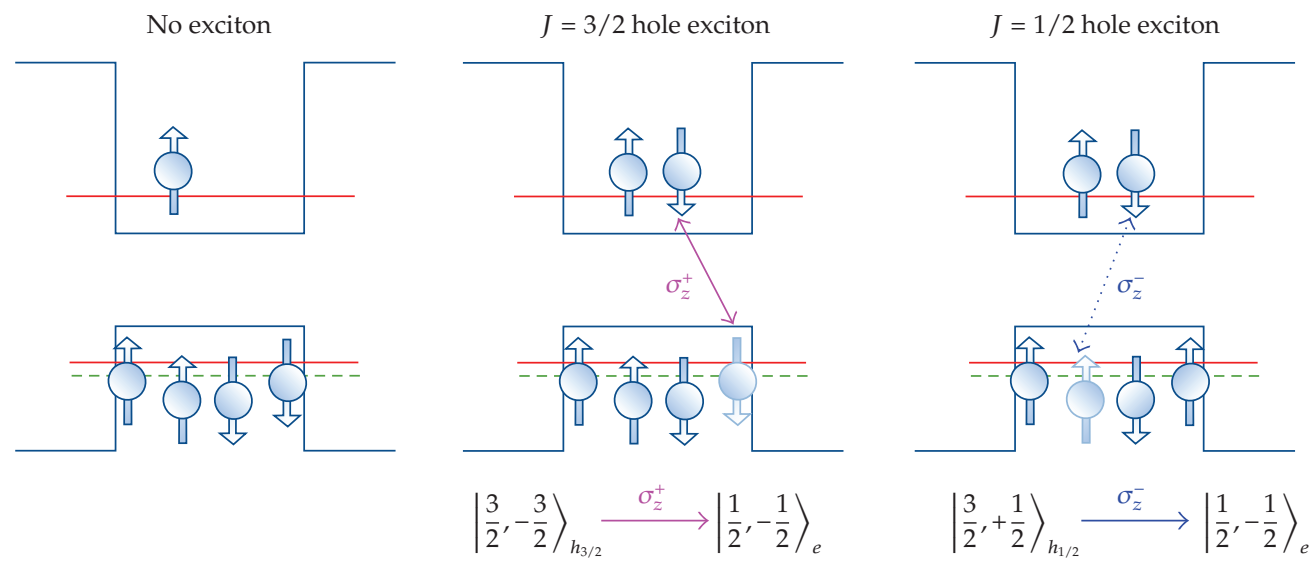

Figure 3: Dipole selection rules in the quantum network nodes.

\subsection{Entanglement Process}

From dipole selection rules, it can easily be shown that the coupling strengths associated with $e-h_{1 / 2}$ and $e-h_{3 / 2}$ excitons can be expressed as $g_{1 / 2}=\sqrt{(1 / 3)} g_{3 / 2}$. This unbalance in the transition strengths between the $e-h_{1 / 2}$ and $e-h_{3 / 2}$ excitons is what makes possible both the process of mapping out quantum information from the storage qubit onto the traveling qubit and creating entanglement between them. How? Because linearly polarized light is nothing but a balanced superposition of right- and left-hand circular polarized light such that $\left|\psi_{p}\right\rangle=$ $|\uparrow\rangle=\left(\left|\sigma_{z}^{+}\right\rangle+\left|\sigma_{z}^{-}\right\rangle\right) / \sqrt{2}$, the right and left circular components of the polarization accumulate different phases due to the unbalance in transition strengths resulting in the rotation of the single-photon linear polarization. This is referred to as the single-photon Faraday Effect [9].

As a result, if the spin of the excess electron is initialized to $|\uparrow\rangle$, then the single-photon polarization rotates in a right-hand circular motion since the right circular polarization component accumulates a larger phase while interacting with $\left|h_{3 / 2}\right\rangle$ valence states as depicted in Figure 3. However, if the spin of the excess electron is initialized to $|\downarrow\rangle$, then the single photon polarization rotates a left-hand circular motion. This Pauli blocking mechanism resulting in the conditional rotation of the single photon linear polarization based on the state of the excess electron spin can effectively be used to encode or map quantum state from the storage qubit onto the traveling qubit or even for creating entanglement between them.

\subsection{Creating Entanglement}

Quantum entanglement is a well-established quantum property that has no counterpart in classical physics; it occurs when the total wave function of the mixed system cannot be written in any basis, as a direct product of independent substates (i.e., tensor product). As a result, the system of the two entangled qubits individually represented, for instance, by states $|\mathbf{0}\rangle$ and $|\mathbf{1}\rangle$ has four new computational basis states designated $|\mathbf{0 0}\rangle,|\mathbf{0 1}\rangle,|\mathbf{1 0}\rangle$, and $|\mathbf{1 1}\rangle$. In the quantum network node, in order to create entanglement, the spin state of a single excess electron in the conduction band of the QD must be initialized to $\left|\psi_{e}\right\rangle=(1 / \sqrt{ } 2)(|\uparrow\rangle+|\downarrow\rangle)$; this is depicted in Figure 4. 


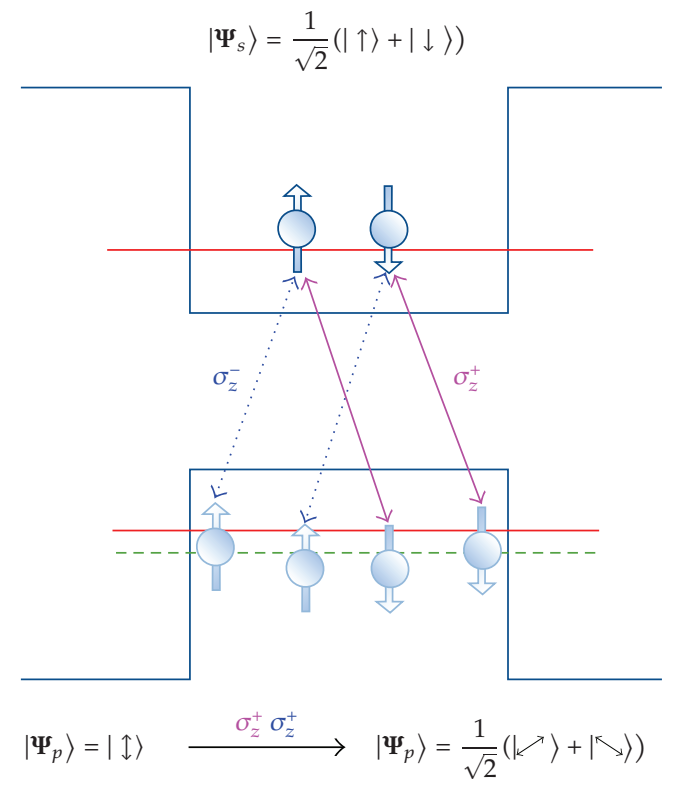

Figure 4: Spin state initialization for entanglement.

Under these conditions, it is unclear in which direction does the polarization of the single photon rotates. The entanglement between the QD excess electron spin and the single photon polarization is predicted to be the greatest at what would correspond to a 45-degree rotation of the linear polarization of the single photon or $\varphi=\pi / 4$ [3]. Transforming to a Bell state eigenbasis for the storage qubit (electron spin) and traveling qubit (photon), we thus have the following maximally entangled Bell state:

$$
\begin{aligned}
\left|\psi_{e p}\right\rangle & =e^{i S_{o}^{h_{3} / 2}}\left|\psi_{X_{3 / 2}}\right\rangle+e^{i s_{o}^{h_{1 / 2}}}\left|\psi_{X_{1 / 2}}\right\rangle \\
& =e^{i S_{o}^{h_{3 / 2}}} \frac{1}{2}\left(\left|\boldsymbol{\sigma}^{+}\right\rangle|\uparrow\rangle+\left|\sigma^{-}\right\rangle|\downarrow\rangle\right)+e^{i s_{o}^{h_{1 / 2}}} \frac{1}{2}\left(\left|\boldsymbol{\sigma}^{-}\right\rangle|\uparrow\rangle+\left|\boldsymbol{\sigma}^{+}\right\rangle|\downarrow\rangle\right) \\
& =e^{-i \theta} \frac{\left(\left|\swarrow^{\nearrow}\right\rangle|\uparrow\rangle \cdot e^{-i \varphi}+|\searrow\rangle|\downarrow\rangle \cdot e^{i \varphi}\right)}{\sqrt{2}} .
\end{aligned}
$$

\section{Modeling Quantum Network Nodes}

The density matrix formalism [10, 11], which is an elegant formulation of quantum mechanics, is used for the modeling of our quantum network. Most importantly, using the density matrix formalism to describe a composite quantum system such as quantum network nodes is indispensable for the analysis of quantum entanglement. This is because the density matrix is able to describe correlations between observables in the various subsystems unlike the Schrodinger's equation formalism. For instance, the entanglement of two qubits forming a composite system represented by the density matrix $\widehat{\rho}$ can be computed directly as either the Von Neumann entropy [12] or the normalized linear entropy [13]. So in order to study 


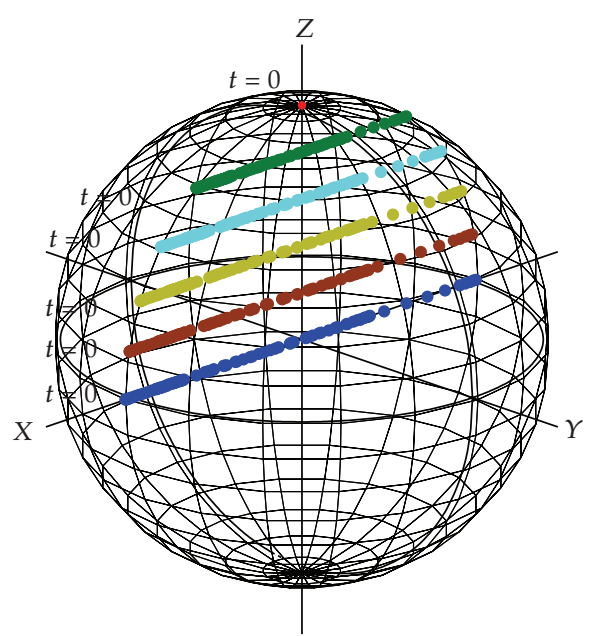

(a)
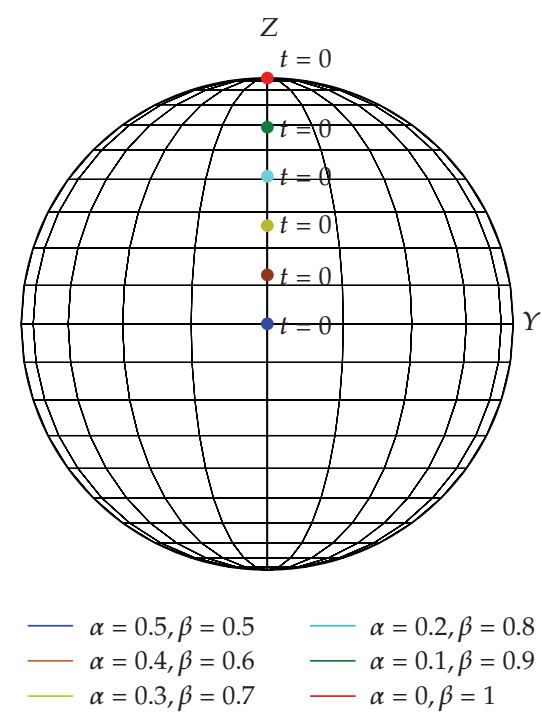

(c)

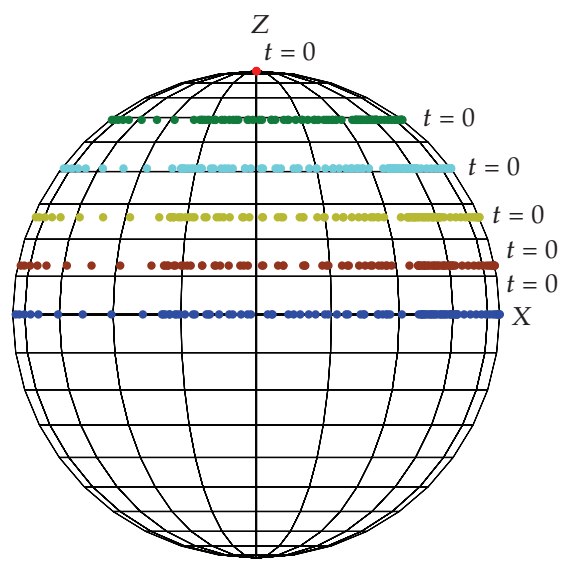

(b)

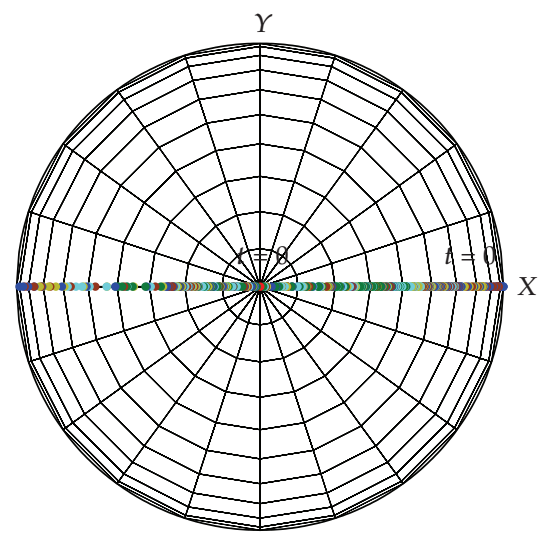

$$
\begin{aligned}
\alpha & =0.5, \beta=0.5 & -\alpha & =0.2, \beta=0.8 \\
\alpha & =0.4, \beta=0.6 & -\alpha & =0.1, \beta=0.9 \\
\alpha & =0.3, \beta=0.7 & -\alpha & =0, \beta=1
\end{aligned}
$$

(d)

Figure 5: Excess electron spin dynamics on the bloch sphere for small $\Delta$.

the dynamics of the entanglement between qubits inside a quantum network node, all that is needed is the time evolution of the density matrix describing the subsystems making up the quantum network node, which is obtained by setting up properly an equation of motion and then solving for it. This is often referred to as the Louiville or Von Neumann Equation of motion for the density matrix, and it is shown in

$$
\frac{d}{d t} \widehat{\boldsymbol{\rho}}=-\frac{i}{\hbar}[\widehat{\mathbf{H}}, \widehat{\boldsymbol{\rho}}]-\frac{1}{2}\{\widehat{\boldsymbol{\Gamma}}, \widehat{\boldsymbol{\rho}}\}
$$




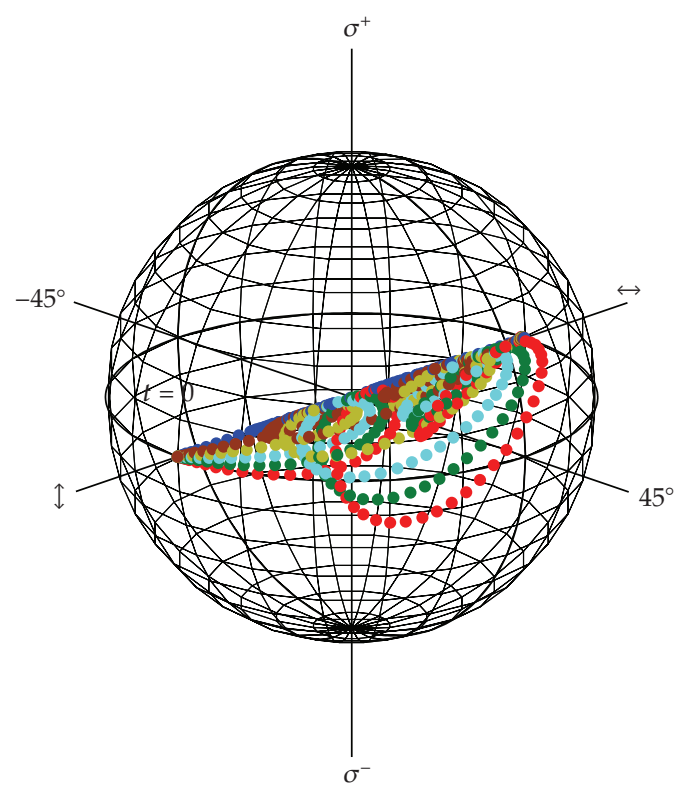

(a)
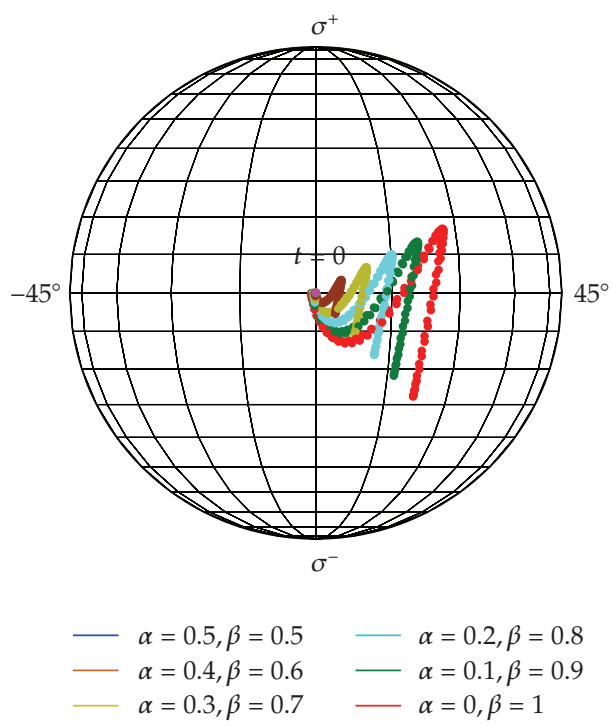

(c)

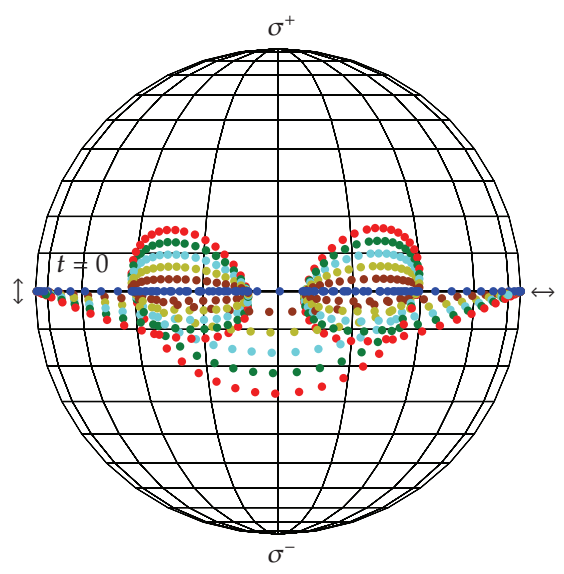

(b)

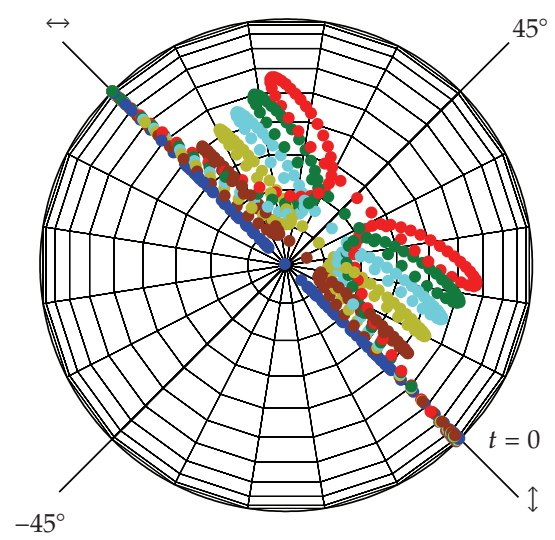

$\begin{aligned} & \alpha=0.5, \beta=0.5 \quad \longrightarrow \quad \alpha=0.2, \beta=0.8 \\ &-\alpha=0.4, \beta=0.6 \quad-\quad \alpha=0.1, \beta=0.9 \\ & \alpha=0.3, \beta=0.7 \quad-\alpha=0, \beta=1\end{aligned}$

(d)

Figure 6: Photon polarization dynamics on the poincare sphere for small $\Delta$.

\subsection{Master Equation}

The Louiville or Von Neumann Equation is the most general form of a master equation for a system whose states are described in term of a density matrix $\widehat{\boldsymbol{\rho}}$ and whose interactions are described according to the Hamiltonian matrix $\widehat{\mathbf{H}}$. However, because relaxation processes are more complicated than just the anticommutator of a single relaxation matrix $\widehat{\Gamma}$ with 
the density matrix $\widehat{\rho}$, we use the following master equation, which is more suitable for the modeling of our quantum network using two relaxation matrices $\widehat{\mathbf{W}}$ and $\widehat{\gamma}$,

$$
\frac{d \rho_{m m^{\prime}}}{d t}=-\frac{i}{\hbar} \sum_{k}\left(H_{m k} \rho_{k m^{\prime}}-\rho_{m k} H_{k m^{\prime}}\right)+\delta_{m m^{\prime}} \sum_{k \neq m} \rho_{k k} W_{m k}-\gamma_{m m^{\prime}} \rho_{m m^{\prime}}
$$

where $W_{m k}$ are transitions rates affecting the diagonal elements of the density matrix and $\gamma_{m m^{\prime}}$ are decoherence rates affecting both diagonal and off-diagonal elements of the density matrix. The matrix $\widehat{\rho}$ is an $N \times N$ matrix with $m=1,2, \ldots, N$ and $m^{\prime}=1,2, \ldots, N$.

\subsection{Matrix Transformations}

Our problem is currently expressed in terms of the subsequent matrix equation

$$
\left[\widehat{\boldsymbol{\rho}}^{\prime}\right]=[\widehat{\mathbf{H}}][\widehat{\boldsymbol{\rho}}]-[\widehat{\boldsymbol{\rho}}][\widehat{\mathbf{H}}]+\delta \sum[\widehat{\boldsymbol{\rho}}][\widehat{\mathbf{W}}]-[\widehat{\boldsymbol{\gamma}}][\widehat{\boldsymbol{\rho}}]
$$

where $\delta \sum[\widehat{\boldsymbol{\rho}}][\widehat{\mathbf{W}}]=\delta_{m m^{\prime}} \sum_{k \neq m} \boldsymbol{\rho}_{k k} W_{m k}$, and $[\widehat{\boldsymbol{\gamma}}][\widehat{\boldsymbol{\rho}}]=(\widehat{\boldsymbol{\gamma}} \cdot \widehat{\boldsymbol{\rho}})_{m m^{\prime}}=\gamma_{m m^{\prime}} \cdot \rho_{m m^{\prime}}$. However, the Runge-Kutta algorithm we are using to solve this system of 1st-order Ordinary Differential Equations numerically requires that the problem is expressed in the following form:

$$
\left[y^{\prime}\right]=[\widehat{\mathcal{L}}][y] .
$$

Equation (3.3) must be rewritten in the form of (3.4). This means rearranging the density matrix $\widehat{\rho}$ into a column vector $y$, and the commutator $[\widehat{\mathbf{H}}, \cdot]+\widehat{\mathbf{W}}-\widehat{\gamma}$ into a matrix $\widehat{\mathcal{L}}$ such that

$$
\begin{gathered}
{[\widehat{\boldsymbol{\rho}}] \stackrel{T_{\mathrm{L}}}{\longrightarrow}\left[y_{[1],\left[\mathrm{mm}^{\prime}\right]}\right],} \\
{\left[[\widehat{\mathbf{H}}, \cdot]_{m m^{\prime}}+\widehat{\mathbf{W}}-\widehat{\boldsymbol{\gamma}}\right] \stackrel{T_{\mathrm{L}}}{\longrightarrow}\left[\widehat{\mathcal{L}}_{\left[m m^{\prime}\right],\left[n n^{\prime}\right]}\right] .}
\end{gathered}
$$

These operations amount to a transformation to Louiville space where $\widehat{\mathbf{L}}_{\left[m m^{\prime}\right],\left[n n^{\prime}\right]}$, is a superoperator (tetradic matrices) acting on that space with dimension $N^{2}$. Equation (3.6) can be broken down into the following three matrix transformations

$$
\begin{gathered}
{\left[[\widehat{\mathbf{H}}, \bullet]_{m m^{\prime}}\right] \stackrel{T_{\mathrm{L}}}{\longrightarrow}\left[\widehat{\mathcal{H}}_{\left[m m^{\prime}\right],\left[n n^{\prime}\right]}\right],} \\
{\left[\widehat{\mathbf{W}}_{m m^{\prime}}\right] \stackrel{T_{\mathrm{L}}}{\longrightarrow}\left[\widehat{\boldsymbol{\Gamma}}_{1\left[m m^{\prime}\right],\left[n n^{\prime}\right]}\right],} \\
{\left[\widehat{\boldsymbol{r}}_{m m^{\prime}}\right] \stackrel{T_{\mathrm{L}}}{\longrightarrow}\left[\widehat{\boldsymbol{\Gamma}}_{2\left[m m^{\prime}\right],\left[n n^{\prime}\right]}\right],}
\end{gathered}
$$

such that

$$
\widehat{\mathcal{L}}_{\left[m m^{\prime}\right],\left[n n^{\prime}\right]}=\widehat{\mathcal{H}}_{\left[m m^{\prime}\right],\left[n n^{\prime}\right]}+\widehat{\boldsymbol{\Gamma}}_{1\left[m m^{\prime}\right],\left[n n^{\prime}\right]}+\widehat{\boldsymbol{\Gamma}}_{2\left[m m^{\prime}\right],\left[n n^{\prime}\right]} .
$$




\subsection{Algorithms}

The first operation transforming $\widehat{\boldsymbol{\rho}}_{m m^{\prime}}$ to $y_{[1],\left[\mathrm{mm}^{\prime}\right]}$ is quite straightforward for an arbitrary size square matrix. The essence of the algorithm is to create a column vector $y$ whose number of elements is $N \times N$ and then fill it up using one row of the density matrix at the time.

The second operation transforming an arbitrary size square matrix $[\widehat{\mathbf{H}}, \bullet]_{m m^{\prime}}$ to $\widehat{\mathcal{H}}_{\left[m m^{\prime}\right],\left[n n^{\prime}\right]}$ is more complex. Before generating algorithms for problems of arbitrary size, it is useful to work out a simple example. Let assume that the density $\widehat{\rho}$ and Hamiltonian $\widehat{\mathbf{H}}$ matrices are $2 \times 2$ matrices so that $m=1,2$ and $m^{\prime}=1,2$ and therefore $N=2$

$$
\widehat{\boldsymbol{\rho}}=\left[\begin{array}{ll}
\rho_{11} & \rho_{12} \\
\rho_{21} & \rho_{22}
\end{array}\right], \quad \widehat{\mathbf{H}}=\left[\begin{array}{ll}
H_{11} & H_{12} \\
H_{21} & H_{22}
\end{array}\right] .
$$

The commutator of the Hamiltonian and the density matrix in matrix form is

$$
[\widehat{\mathbf{H}}, \widehat{\boldsymbol{\rho}}]=\left[\begin{array}{cc}
{\left[H_{12} \rho_{21}-H_{21} \rho_{12}\right]} & {\left[\begin{array}{c}
H_{11} \rho_{12}-H_{12} \rho_{22} \\
-H_{12} \rho_{11}-H_{22} \rho_{12}
\end{array}\right]} \\
{\left[\begin{array}{c}
H_{21} \rho_{11}-H_{22} \rho_{21} \\
-H_{11} \rho_{21}-H_{21} \rho_{22}
\end{array}\right]} & {\left[H_{21} \rho_{12}-H_{12} \rho_{21}\right]}
\end{array}\right] .
$$

Next, the flowing operation $[\widehat{\mathbf{H}}, \cdot]_{2 \times 2} \rightarrow[\widehat{\mathcal{H}}]_{4 \times 4}$ is performed on the Hamiltonian $\widehat{\mathbf{H}}$ such that $(d / d t)[\widehat{\boldsymbol{\rho}}]_{m m^{\prime}}=(-(i / \hbar))[\widehat{\mathbf{H}}, \widehat{\boldsymbol{\rho}}]_{m m^{\prime}} \rightarrow(d / d t) y_{[1],\left[m m^{\prime}\right]}=\widehat{\mathcal{H}}_{\left[m m^{\prime}\right],\left[n n^{\prime}\right]} y_{[1],\left[m m^{\prime}\right]}$,

$$
\left[\begin{array}{cc}
H_{11} & H_{12} \\
H_{21} & H_{22}
\end{array}\right] \longrightarrow\left[\begin{array}{cccc}
0 & -H_{21} & H_{12} & 0 \\
-H_{12} & H_{11}-H_{22} & 0 & H_{12} \\
H_{21} & 0 & H_{22}-H_{11} & -H_{21} \\
0 & H_{21} & -H_{12} & 0
\end{array}\right] .
$$

The algorithm for an arbitrary square matrix $\widehat{\mathcal{H}}$ of size $N^{2}$ by $N^{2}$ is considered next. First, we need to define new indices $i$ and $j$ which are used to identify sub-block matrices. So (3.13) becomes

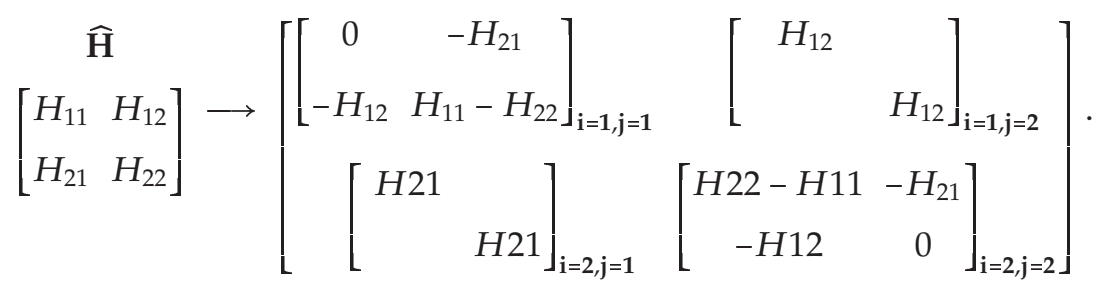


The strategy adopted to fill this matrix up consists of dividing the matrix $\widehat{\mathcal{H}}$ into smaller quadrants or matrices $\widehat{\mathrm{L}}_{i j}$ of size $N \times N$

$$
\widehat{\mathcal{H}}=\left[\begin{array}{cccc}
{\left[\widehat{\mathbf{L}}_{11}\right]} & {\left[\widehat{\mathbf{L}}_{12}\right]} & \cdots & {\left[\widehat{\mathbf{L}}_{1 m^{\prime}}\right]} \\
{\left[\widehat{\mathbf{L}}_{21}\right]} & \ddots & & \\
\vdots & & \ddots & \\
{\left[\widehat{\mathbf{L}}_{m 1}\right]} & & & {\left[\widehat{\mathbf{L}}_{m m^{\prime}}\right]}
\end{array}\right] .
$$

The off-diagonal matrices $\widehat{\mathbf{L}}_{i j}(i \neq j)$ identified with the indices $i$ and $j$ are filled with element from the original Hamiltonian $[\widehat{\mathbf{H}}, \cdot]_{m m^{\prime}}$ using the same indices $i$ and $j$ such that matrices $\widehat{\mathbf{L}}_{i j}$ are themselves diagonal matrices filled with elements $H_{i j}$ of $\widehat{\mathbf{H}}$. Therefore

$$
\widehat{\mathbf{L}}_{i \neq j}=\left[\begin{array}{cccc}
H_{i j} & 0 & 0 & 0 \\
0 & H_{i j} & 0 & 0 \\
0 & 0 & \ddots & 0 \\
0 & 0 & 0 & H_{i j}
\end{array}\right] .
$$

As for the diagonal matrices $\left(\widehat{\mathbf{L}}_{i j}(i=j)\right)$, they are constructed such that

$$
\widehat{\mathbf{L}}_{i=j}=\left[\begin{array}{ccccc}
H_{i j}-H_{1,1} & -H_{1,2} & -H_{1,3} & \cdots & -H_{1, m^{\prime}} \\
-H_{2,1} & H_{i j}-H_{2,2} & -H_{2,3} & \cdots & -H_{2, m^{\prime}} \\
-H_{3,1} & -H_{3,2} & H_{i j}-H_{3,3} & \ddots & -H_{3, m^{\prime}} \\
\vdots & \vdots & \ddots & \ddots & \vdots \\
-H_{m, 1} & -H_{m, 2} & -H_{m, 3} & \cdots & H_{i j}-H_{m, m^{\prime}}
\end{array}\right] .
$$

Finally, the relaxation matrices $\widehat{\mathbf{W}}$ and $\widehat{r}$ also need to be transformed. In the original master equation, $\widehat{\mathbf{W}}$ and $\widehat{\gamma}$ are $N \times N$ matrices and are written as

$$
\begin{aligned}
\widehat{\mathbf{W}}_{m m^{\prime}} & =\left[\begin{array}{ccccc}
W_{11} & W_{12} & W_{13} & \cdots & W_{1 m^{\prime}} \\
W_{21} & W_{22} & W_{23} & \cdots & W_{2 m^{\prime}} \\
W_{31} & W_{32} & W_{33} & \ddots & \vdots \\
\vdots & \vdots & \ddots & \ddots & W_{(m-1) m^{\prime}} \\
W_{m 1} & W_{m 2} & \cdots & W_{m\left(m^{\prime}-1\right)} & W_{m m^{\prime}}
\end{array}\right], \\
\widehat{\gamma}_{m m^{\prime}} & =\left[\begin{array}{ccccc}
\gamma_{11} & \gamma_{12} & \gamma_{13} & \cdots & \gamma_{1 m^{\prime}} \\
\gamma_{21} & \gamma_{22} & \gamma_{23} & \cdots & \gamma_{2 m^{\prime}} \\
\gamma_{31} & \gamma_{32} & \gamma_{33} & \ddots & \vdots \\
\vdots & \vdots & \ddots & \ddots & \gamma_{(m-1) m^{\prime}} \\
\gamma_{m 1} & \gamma_{m 2} & \cdots & \gamma_{m\left(m^{\prime}-1\right)} & \gamma_{m m^{\prime}}
\end{array}\right],
\end{aligned}
$$


First, $\widehat{\boldsymbol{\Gamma}}_{1}$ matrix is considered. It is built both from relaxation matrices $\widehat{\mathbf{W}}$ and $\widehat{\gamma}$ in connection with the diagonal elements of the density matrix. When $m=m^{\prime}$, the term relating to the transition rates in (3.2) in matrix form is written as

$$
\begin{aligned}
\delta \sum[\widehat{\boldsymbol{\rho}}][\widehat{\mathbf{W}}] & =\delta_{m m^{\prime}} \sum_{k \neq m} \rho_{k k} W_{m k} \\
& =\left[\begin{array}{cccc}
\sum_{k} \rho_{k k} W_{1 k} & 0 & \cdots & 0 \\
0 & \sum_{k} \rho_{k k} W_{2 k} & 0 & 0 \\
\vdots & \vdots & \ddots & \vdots \\
0 & 0 & \cdots & \sum_{k} \rho_{k k} W_{m k}
\end{array}\right],
\end{aligned}
$$

whereas the term relating to the decoherence rates in (3.2) in matrix form is written

$$
\begin{aligned}
{[\widehat{\boldsymbol{\gamma}}][\widehat{\boldsymbol{\rho}}] } & =\gamma_{m m} \rho_{m m} \\
& =\rho_{m m} \sum_{k} W_{k m} \\
& =\left[\begin{array}{cccc}
\rho_{11} \sum_{k} W_{\mathrm{k} 1} & 0 & \cdots & 0 \\
0 & \rho_{22} \sum_{k} W_{k 2} & 0 & 0 \\
\vdots & \vdots & \ddots & \vdots \\
0 & 0 & \cdots & \rho_{k k} \sum_{k} W_{k m}
\end{array}\right],
\end{aligned}
$$

since

$$
\gamma_{m m}=\frac{1}{2} \sum_{k}\left(W_{k m}+W_{k m^{\prime}}\right)=\frac{1}{2} \sum_{k}\left(W_{k m}+W_{k m}\right)=\sum_{k \neq m} W_{k m}
$$

Combining (3.19) and (3.20), we get the following new term where $\widehat{\mathbf{W}}$ and $\widehat{\gamma}$ are forming the matrix $\widehat{\mathbf{W}}^{\prime}$,

$$
\begin{aligned}
{[\widehat{\boldsymbol{\rho}}]\left[\widehat{\mathbf{W}}^{\prime}\right] } & =\delta \sum[\widehat{\boldsymbol{\rho}}][\widehat{\mathbf{W}}]-[\widehat{\boldsymbol{\gamma}}][\widehat{\boldsymbol{\rho}}] \\
& =\delta_{m m \prime} \sum_{k \neq m} \rho_{k k} W_{m k}-\rho_{m m} \sum_{k} W_{k m} \\
& =\left[\begin{array}{ccll}
\sum_{k} \rho_{k k} W_{1 k}-\rho_{11} \sum_{k} W_{k 1} & 0 & \cdots & 0 \\
0 & \sum_{k} \rho_{k k} W_{2 k}-\rho_{22} \sum_{k} W_{k 2} & 0 & 0 \\
\vdots & \vdots & \ddots & \vdots \\
0 & 0 & \cdots \sum_{k} \rho_{k k} W_{m k}-\rho_{k k} \sum_{k} W_{k m}
\end{array}\right] .
\end{aligned}
$$


Before coming with an algorithm that can transform $\widehat{\mathbf{W}}^{\prime}$ to $\widehat{\boldsymbol{\Gamma}}_{1}$ for a problem of arbitrary size, it is useful to work out a simple example. It is assumed for the moment that $\widehat{\boldsymbol{\rho}}, \widehat{\mathbf{W}}$, and $\widehat{\gamma}$ are $2 \times 2$ matrices such that $m=1,2$ and $m^{\prime}=1,2$ and therefore $N=2$

$$
\widehat{\boldsymbol{\rho}}=\left[\begin{array}{ll}
\rho_{11} & \rho_{12} \\
\rho_{21} & \rho_{22}
\end{array}\right], \quad \widehat{\mathbf{W}}=\left[\begin{array}{ll}
W_{11} & W_{12} \\
W_{21} & W_{22}
\end{array}\right], \quad \widehat{\boldsymbol{\gamma}}=\left[\begin{array}{ll}
\gamma_{11} & \gamma_{12} \\
\gamma_{21} & \gamma_{22}
\end{array}\right]=\left[\begin{array}{cc}
W_{21} & 0 \\
0 & W_{12}
\end{array}\right] .
$$

From the master equation, the terms related to transition and decoherence rates are therefore expressed as

$$
\begin{aligned}
\delta \sum[\hat{\boldsymbol{\rho}}][\widehat{\mathbf{W}}]-[\widehat{\boldsymbol{\gamma}}][\hat{\boldsymbol{\rho}}] & =\delta_{m m^{\prime}} \sum_{k \neq m} \rho_{k k} W_{m k}-\rho_{m m} \sum_{k} W_{k m} \\
& =\left[\begin{array}{cc}
\rho_{22} W_{12}-\rho_{11} W_{21} & 0 \\
0 & \rho_{11} W_{21}-\rho_{22} W_{12}
\end{array}\right] .
\end{aligned}
$$

In Louiville space, the term $\delta \sum[\widehat{\boldsymbol{\rho}}][\widehat{\mathbf{W}}]-[\widehat{\boldsymbol{\gamma}}][\widehat{\boldsymbol{\rho}}]$ is rewritten $y_{[1],[4]}^{\mathrm{T}} \widehat{\boldsymbol{\Gamma}}_{1[4],[4]}$ such that

$$
\begin{aligned}
& y^{\mathrm{T}}(1, J) \quad \widehat{\boldsymbol{\Gamma}}_{1}(I, J) \\
& {\left[\begin{array}{llll}
\rho_{11} & 0 & 0 & \rho_{22}
\end{array}\right]\left[\begin{array}{cccc}
-W_{21} & 0 & 0 & W_{21} \\
0 & 0 & 0 & 0 \\
0 & 0 & 0 & 0 \\
W_{12} & 0 & 0 & -W_{12}
\end{array}\right] .}
\end{aligned}
$$

Thus, in order to obtain $\widehat{\Gamma}_{1}$ for an arbitrary size problem, the strategy is to create a square matrix of size $N^{2} \times N^{2}$ such that the term $\delta \sum[\widehat{\boldsymbol{\rho}}][\widehat{\mathbf{W}}]-[\widehat{\boldsymbol{r}}][\widehat{\boldsymbol{\rho}}]$ is rewritten $\boldsymbol{y}_{[1],\left[\mathrm{mm}^{\prime}\right]}^{\mathrm{T}} \widehat{\boldsymbol{\Gamma}}_{1\left[\mathrm{~mm}^{\prime}\right],\left[\mathrm{nn}^{\prime}\right]}$, and then fill $\widehat{\Gamma}_{1}$. The notation for matrix indices in state space is as follows $i=1,2, \ldots, N$ and $j=1,2, \ldots, N$. Their counterparts in Louiville space are $I=1,2, \ldots, N^{2}$ and $J=1,2, \ldots, N^{2}$. The key is to identify the columns (or J's) in $\widehat{\Gamma}_{1}$ that needed to be filled, and then fill out the appropriate rows (or I's) with the appropriate rates. The columns or $J$ 's to be filled are the ones whose indices are matching the $J^{\prime}$ s in $y^{\mathrm{T}}(1, J)$ that correspond to diagonal terms in the original density matrix $\widehat{\boldsymbol{\rho}}_{m m^{\prime}}$, namely, $\rho_{m=1, m^{\prime}=1} \rightarrow y_{1, J=1}$ and $\rho_{m=2, m^{\prime}=2} \rightarrow y_{1, J=4}$ as shown in the example above. A general expression for the columns to be filled is

$$
J=(j-1) \cdot N+j, \quad \text { where } j=1,2, \ldots, N .
$$

It turns out that the set of indices for rows and columns to be filled are identical $(I=J)$; therefore determining the indices for the columns to be filled also give the ones for the rows.

Therefore, the algorithm to generate elements of $\widehat{\boldsymbol{\Gamma}}_{1}$ for a problem of an arbitrary size is as follows

$$
\widehat{\boldsymbol{\Gamma}}_{1}(I, J)= \begin{cases}\widehat{\mathbf{W}}(i, j) & (\text { for } I \neq J), \\ -\widehat{\gamma}(i, j) & (\text { for } I=J),\end{cases}
$$


where $I=(i-1) \cdot N+i$ and $J=(j-1) \cdot N+j$ with $i=1,2, \ldots, N$ and $j=1,2, \ldots, N$. In Louiville space, when considering only the diagonal terms of the density matrix, $\widehat{\Gamma}_{1}$ can be written as

$$
\widehat{\mathbf{W}}=\left[\begin{array}{ccccc}
-\sum_{k \neq 1} W_{k 1} & W_{21} & W_{31} & \ldots & W_{k 1} \\
W_{12} & -\sum_{k \neq 2} W_{k 2} & W_{32} & \ldots & W_{k 2} \\
W_{13} & W_{23} & -\sum_{k \neq 3} W_{\mathrm{k} 3} & \ddots & \vdots \\
\vdots & \vdots & \ddots & \ddots & W_{k(m-1)} \\
W_{1 m} & W_{2 m} & \ldots & W_{(k-1) m} & -\sum_{k \neq m} W_{k m}
\end{array}\right] .
$$

Next, $\widehat{\Gamma}_{2}$ is considered. Unlike $\widehat{\Gamma}_{1}, \widehat{\Gamma}_{2}$ is associated with the case when $m \neq m^{\prime}$ in the master equation see (3.3). This means that $\delta \sum[\widehat{\boldsymbol{\rho}}][\widehat{\mathbf{W}}]=0$; therefore, only $-[\widehat{\boldsymbol{\gamma}}][\widehat{\boldsymbol{\rho}}]$ contributes to the master equation. Furthermore, $\widehat{\Gamma}_{2}$ is generated from only the decoherence rates $\gamma_{m m^{\prime}}$, which are off-diagonal elements of $\widehat{\gamma}$ such that

$$
\widehat{\Upsilon}_{m \neq m^{\prime}}=\frac{1}{2} \sum_{k}\left(W_{m k}+W_{k m^{\prime}}\right)
$$

These rates are included in the master equation by means of a Hadamard or Schur product between the relaxation matrix $\widehat{\gamma}$ and the density matrix $\widehat{\rho}$ such that

$$
\begin{aligned}
{[\widehat{\boldsymbol{\gamma}}][\boldsymbol{\rho}] } & =\gamma_{m m^{\prime}} \cdot \rho_{m m^{\prime}} \\
& =\left[\begin{array}{ccccc}
0 & \gamma_{12} \rho_{12} & \gamma_{13} \rho_{13} & \ldots & \gamma_{1 m^{\prime}} \rho_{1 m^{\prime}} \\
\gamma_{21} \rho_{21} & 0 & \gamma_{23} \rho_{23} & \ldots & \gamma_{2 m^{\prime}} \rho_{2 m^{\prime}} \\
\gamma_{31} \rho_{31} & \gamma_{32} \rho_{32} & 0 & \ddots & \vdots \\
\vdots & \vdots & \ddots & \ddots & \gamma_{(m-1) m^{\prime}} \rho_{(m-1) m^{\prime}} \\
\gamma_{m 1} \rho_{m 1} & \gamma_{m 2} \rho_{m 2} & \cdots & \gamma_{m\left(m^{\prime}-1\right)} \rho_{m\left(m^{\prime}-1\right)} & 0
\end{array}\right] .
\end{aligned}
$$

Before coming with an algorithm that can transform $\widehat{\gamma}$ to $\widehat{\Gamma}_{1}$ for a problem of arbitrary size, it is useful to work out a simple example. It is assumed for the moment that $\widehat{\rho}$ and $\widehat{\gamma}$ are $2 \times 2$ matrices such that $m=1,2$ and $m^{\prime}=1,2$ and therefore $N=2$

$$
\begin{gathered}
\widehat{\boldsymbol{\rho}}=\left[\begin{array}{ll}
\rho_{11} & \rho_{12} \\
\rho_{21} & \rho_{22}
\end{array}\right], \\
\widehat{\boldsymbol{r}}_{i \neq j}=\left[\begin{array}{cc}
0 & \gamma_{12} \\
\gamma_{21} & 0
\end{array}\right]=\left[\begin{array}{cc}
0 & W_{12} \\
W_{21} & 0
\end{array}\right] .
\end{gathered}
$$


From the master equation, the terms related to decoherence rates are therefore expressed as

$$
[\widehat{\boldsymbol{\gamma}}][\hat{\boldsymbol{\rho}}]=\gamma_{m m^{\prime}} \cdot \rho_{m m^{\prime}}=\left[\begin{array}{cc}
0 & \rho_{12} W_{12} \\
\rho_{21} W_{21} & 0
\end{array}\right] .
$$

In Louiville space, the term $[\widehat{\boldsymbol{r}}][\widehat{\boldsymbol{\rho}}]$ is rewritten $\widehat{\boldsymbol{\Gamma}}_{2[4],[4]} y_{[1],[4]}$ such that

$$
\begin{aligned}
& \widehat{\boldsymbol{\Gamma}}_{2}(I, J) y \quad \gamma(I, 1) \\
& {\left[\begin{array}{cccc}
0 & 0 & 0 & 0 \\
0 & \gamma_{12} & 0 & 0 \\
0 & 0 & \gamma_{21} & 0 \\
0 & 0 & 0 & 0
\end{array}\right]\left[\begin{array}{l}
\rho_{11} \\
\rho_{12} \\
\rho_{21} \\
\rho_{22}
\end{array}\right] .}
\end{aligned}
$$

Consequently, in order to obtain $\widehat{\Gamma}_{2}$ for an arbitrary size problem, the strategy is to create first a vector $\gamma_{\left[\mathrm{mm}^{\prime}\right],[1]}$ from $\widehat{\gamma}_{m m^{\prime}}$ whose number of elements is $N^{2}$ and then fill it up by concatenating the rows of the density matrix at the time. Next, $\widehat{\Gamma}_{2}$, a square matrix of size $N^{2} \times N^{2}$, is generated by filling its diagonal elements with elements from the vector $\gamma_{\left[\mathrm{mm}^{\prime}\right],[1]}$. Evidently, the matrix $\widehat{\Gamma}_{2}$ always consists of a diagonal matrix. The master equation in (3.3) now takes on the following form:

$$
\left[y^{\prime}\right]=[\widehat{\mathcal{H}}][y]-[y]^{\mathrm{T}}\left[\widehat{\boldsymbol{\Gamma}}_{1}\right]-\left[\widehat{\boldsymbol{\Gamma}}_{2}\right][y]
$$

\subsection{Solving for Entanglement}

The entanglement of two qubits forming a composite system represented by the density matrix $\rho^{A \otimes B}$ can be computed directly as either the Von Neumann entropy [12] or the normalized linear entropy [13] of the reduced density matrix of either of the two qubits. Equations (3.35) below show, respectively, the Von Neumann and normalized linear entropies

$$
\begin{aligned}
& S_{\mathrm{VN}}=-\operatorname{Tr}_{\mathrm{A}}\left[\operatorname{Tr}_{\mathrm{B}} \rho^{A \otimes B} \log _{2}\left(\operatorname{Tr}_{\mathrm{B}} \rho^{A \otimes B}\right)\right], \\
& S_{\mathrm{NL}}=2\left[1-\operatorname{Tr}_{\mathrm{A}}\left(\operatorname{Tr}_{\mathrm{B}} \rho^{A \otimes B}\right)^{2}\right] .
\end{aligned}
$$

Whether it is calculated from the Von Neumann entropy or the normalized linear entropy, the entanglement between two qubits ranges from 0 to 1 , where 1 means that they are maximally entangled. 
Let us consider the "pure" state density matrix describing the combined spin-photon system within a single high- $Q$ cavity shown in (3.36). It contains time-dependent variables $\rho_{i j}$, which describe the probability of occupying each distinct set of states in the cavity system, which are $\left|\downarrow, \sigma_{z}^{-}\right\rangle$, corresponding to the state when the excess electron spin being down with a left circularly polarized (LCP or $\sigma_{z}^{-}$) photon in the cavity, $\left|\downarrow, \sigma_{z}^{+}\right\rangle$to the excess electron spin being down with a right circularly polarized (RCP or $\sigma_{z}^{+}$) photon in the cavity, $\left|\downarrow, \mathbf{X}_{1 / 2}\right\rangle$ to the excess electron spin being down with a $e-h_{1 / 2}$ exciton (trion), $\left|\downarrow, \mathrm{X}_{3 / 2}\right\rangle$ to the excess electron spin being down with a $e-h_{3 / 2}$ exciton (trion), $\left|\uparrow, \sigma_{z}^{-}\right\rangle$to the excess electron spin being up with an LCP photon in the cavity, $\left|\uparrow, \sigma_{\mathrm{z}}^{+}\right\rangle$to the excess electron spin being up with a RCP photon in the cavity, $\left|\uparrow, \mathbf{X}_{1 / 2}\right\rangle$, to the excess electron spin being up with a $e-h_{1 / 2}$ exciton (trion), and $\left|\uparrow, X_{3 / 2}\right\rangle$ to the excess electron spin being up with a $e-h_{3 / 2}$ exciton (trion)

$$
\begin{aligned}
& \widehat{\rho}=|\boldsymbol{\psi}(t)\rangle\langle\boldsymbol{\psi}(t)| \\
& \left\langle\downarrow, \sigma_{z}^{-}\right|\left\langle\downarrow, \sigma_{z}^{+}\right|\left\langle\downarrow, X_{1 / 2}\right|\left\langle\downarrow, X_{3 / 2}\right|\left\langle\uparrow, \sigma_{z}^{-}\right|\left\langle\uparrow, \sigma_{z}^{+}\right|\left\langle\uparrow, X_{1 / 2}\right|\left\langle\uparrow, X_{3 / 2}\right| \\
& =\left[\begin{array}{cccccccc}
\rho_{11} & \rho_{12} & \rho_{13} & \rho_{14} & \rho_{15} & \rho_{16} & \rho_{17} & \rho_{18} \\
\rho_{21} & \rho_{22} & \rho_{23} & \rho_{24} & \rho_{25} & \rho_{26} & \rho_{27} & \rho_{28} \\
\rho_{31} & \rho_{32} & \rho_{33} & \rho_{34} & \rho_{35} & \rho_{36} & \rho_{37} & \rho_{38} \\
\rho_{41} & \rho_{42} & \rho_{43} & \rho_{44} & \rho_{45} & \rho_{46} & \rho_{47} & \rho_{48} \\
\rho_{51} & \rho_{52} & \rho_{53} & \rho_{54} & \rho_{55} & \rho_{56} & \rho_{57} & \rho_{58} \\
\rho_{61} & \rho_{62} & \rho_{63} & \rho_{64} & \rho_{65} & \rho_{66} & \rho_{67} & \rho_{68} \\
\rho_{71} & \rho_{72} & \rho_{73} & \rho_{74} & \rho_{75} & \rho_{76} & \rho_{77} & \rho_{78} \\
\rho_{81} & \rho_{82} & \rho_{83} & \rho_{84} & \rho_{85} & \rho_{86} & \rho_{87} & \rho_{88}
\end{array}\right] \begin{array}{c}
\left|\downarrow, \sigma_{\mathbf{z}}^{-}\right\rangle \\
\left|\downarrow, \boldsymbol{\sigma}_{\mathbf{z}}^{+}\right\rangle \\
\left|\downarrow, \mathbf{X}_{\mathbf{1} 2}\right\rangle \\
\left|\uparrow, \boldsymbol{\sigma}_{\mathbf{z}}\right\rangle \\
\left|\uparrow, \mathbf{X}_{\mathbf{z}}\right\rangle \\
\left|\uparrow, \mathbf{X}_{3 / 2}\right\rangle \\
\end{array}
\end{aligned}
$$

Next, in order to solve for the entanglement dynamics inside our quantum network node, we need first the Hamiltonian, which is obtained in the rotating frame, as well as the relaxation matrices $\widehat{\mathbf{W}}$ and $\widehat{\gamma}$

$$
\begin{aligned}
& \left\langle\downarrow, \sigma_{\mathrm{z}}^{-}\right|\left\langle\downarrow, \sigma_{\mathrm{z}}^{+}\right|\left\langle\downarrow, \mathbf{X}_{1 / 2}\right|\left\langle\downarrow, \mathbf{X}_{3 / 2}\right|\left\langle\uparrow, \sigma_{z}^{-}\right|\left\langle\uparrow, \sigma_{z}^{+}\right|\left\langle\uparrow, \mathbf{X}_{1 / 2}\right|\left\langle\uparrow, \mathbf{X}_{3 / 2}\right|
\end{aligned}
$$

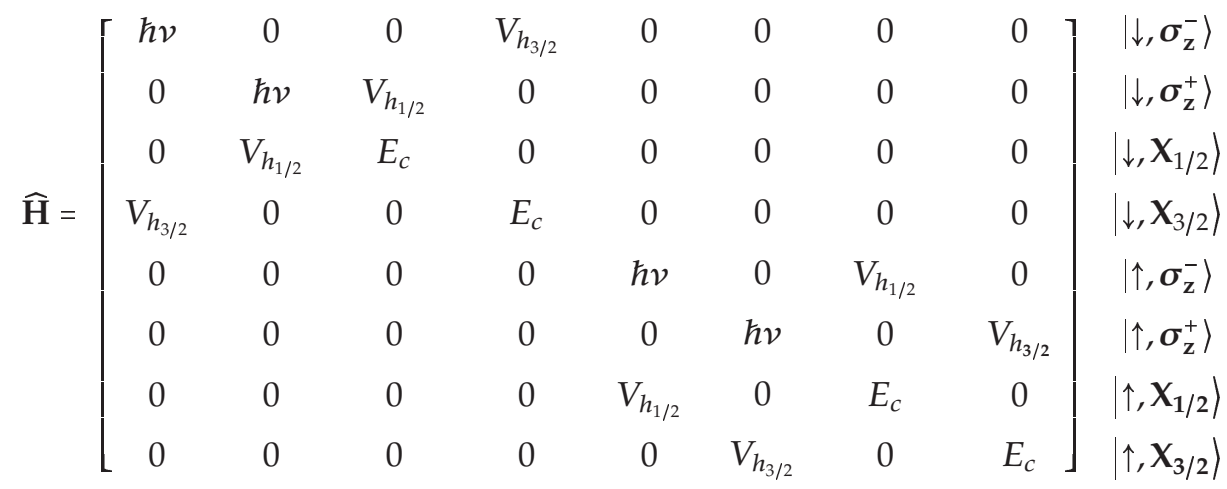


where $E_{\mathrm{c}}$ is the energy of the excess electron, $v$ the photon frequency, $V_{\mathrm{h}_{3 / 2}}=\hbar g_{3 / 2}$ the interaction energy associated with the $e-h_{3 / 2}$ excitons, and $V_{\mathrm{h}_{1 / 2}}=\hbar g_{1 / 2}$ the interaction energy associated with the $e-h_{1 / 2}$ excitons

\begin{tabular}{|c|c|c|c|c|c|c|c|c|}
\hline$\left\langle\downarrow, \sigma_{z}^{-}\right|$ & $\left\langle\downarrow, \sigma_{z}^{+}\right|$ & $\left\langle\downarrow, X_{1 / 2}\right|$ & $\left\langle\downarrow, X_{3 / 2}\right|$ & $\left\langle\uparrow, \sigma_{z}^{-}\right|$ & $\left\langle\uparrow, \sigma_{z}^{+}\right|$ & $\left\langle\uparrow, X_{1 / 2}\right|$ & $\left\langle\uparrow, X_{3 / 2}\right|$ & \\
\hline$\Gamma^{-} W_{15}$ & 0 & 0 & $W_{14}$ & $W_{15}$ & 0 & 0 & 0 & ]$^{\left|\downarrow, \sigma_{z}^{-}\right\rangle}$ \\
\hline 0 & $-W_{26}$ & $W_{23}$ & 0 & 0 & $W_{26}$ & 0 & 0 & $\left.|\downarrow| \downarrow, \sigma_{z}^{+}\right\rangle$ \\
\hline 0 & 0 & $-W_{23}-W_{34}$ & $W_{34}$ & 0 & 0 & $W_{37}$ & $W_{38}$ & $\left|\downarrow, \mathbf{X}_{1 / 2}\right\rangle$ \\
\hline 0 & 0 & $\begin{array}{c}-W_{37}-W_{38} \\
W_{34}\end{array}$ & $\begin{array}{l}-W_{14}-W_{34} \\
-W_{44}-W_{49}\end{array}$ & 0 & 0 & $W_{47}$ & $W_{48}$ & $\left|\downarrow, X_{3 / 2}\right\rangle$ \\
\hline$W_{15}$ & 0 & 0 & $\begin{array}{c}-W_{47}-W_{48} \\
0\end{array}$ & $-W_{15}$ & 0 & $W_{57}$ & 0 & $\left|\uparrow, \sigma_{z}^{-}\right\rangle$ \\
\hline 0 & $W_{26}$ & 0 & 0 & 0 & $-W_{26}$ & 0 & $W_{68}$ & $\left|\uparrow, \sigma_{z}^{+}\right\rangle$ \\
\hline 0 & 0 & $W_{37}$ & $W_{47}$ & 0 & 0 & $\begin{array}{l}-W_{57}-W_{37} \\
-W_{47}-W_{78}\end{array}$ & $W_{78}$ & $\left|\uparrow, X_{1 / 2}\right\rangle$ \\
\hline 0 & 0 & $W_{38}$ & $W_{48}$ & 0 & 0 & $\begin{array}{c}-W_{47}-W_{78} \\
W_{78}\end{array}$ & $\begin{array}{l}-W_{68}-W_{38} \\
-W_{48}-W_{78}\end{array}$ & ]$_{\left|\uparrow, X_{3 / 2}\right\rangle}$ \\
\hline
\end{tabular}

where $W_{23}, W_{14}, W_{57}$, and $W_{68}$ are nonreversible decay rates, respectively, from the trion state $\left|\downarrow, X_{1 / 2}\right\rangle$ to the photonic state $\left\langle\downarrow, \sigma_{z}^{+}\right|$, from the trion state $\left|\downarrow, X_{3 / 2}\right\rangle$ to the photonic state $\left\langle\downarrow, \sigma_{z}^{-}\right|$, from the trion state $\left|\uparrow, \mathbf{X}_{1 / 2}\right\rangle$ to the photonic state $\left\langle\uparrow, \boldsymbol{\sigma}_{\mathbf{z}}^{-}\right|$, and from the trion state $\left|\uparrow, \mathbf{X}_{3 / 2}\right\rangle$ to the photonic state $\left\langle\uparrow, \boldsymbol{\sigma}_{\mathbf{z}}^{+}\right|$. Next, the Fermi contact terms are depicted by $W_{15}$ representing a transition for the excess electron spin from $\left|\downarrow, \sigma_{z}^{-}\right\rangle$to $\left\langle\uparrow, \sigma_{z}^{-}\right|$and $W_{26}$ representing a transition for the excess electron spin from $\left|\downarrow, \sigma_{\mathbf{z}}^{+}\right\rangle$to $\left\langle\uparrow, \boldsymbol{\sigma}_{\mathbf{z}}^{+}\right|$. Last, the hole hyperfine interaction terms are depicted by $W_{37}, W_{48}, W_{34}, W_{78}, W_{38}$, and $W_{47}$. They, respectively, represent the transition from the following trion state $\left|\downarrow, \mathbf{X}_{1 / 2}\right\rangle$ to the trion state $\left\langle\uparrow, \mathbf{X}_{1 / 2}\right|$, the transition from the following trion state $\left|\downarrow, X_{3 / 2}\right\rangle$ to the trion state $\left\langle\uparrow, X_{3 / 2}\right|$, the transition from the following trion state $\left|\downarrow, \mathbf{X}_{1 / 2}\right\rangle$ to the trion state $\left|\downarrow, \mathbf{X}_{3 / 2}\right\rangle$, the transition from the following trion state $\left|\uparrow, \mathbf{X}_{1 / 2}\right\rangle$ to the trion state $\left\langle\uparrow, \mathbf{X}_{3 / 2}\right|$, the transition from the following trion state $\left|\downarrow, \mathbf{X}_{1 / 2}\right\rangle$ to the trion state $\left\langle\uparrow, \mathbf{X}_{3 / 2}\right|$, and the transition from the following trion state $\left|\downarrow, \mathbf{X}_{3 / 2}\right\rangle$ to the trion state $\left|\uparrow, \mathbf{X}_{1 / 2}\right\rangle$

$$
\begin{aligned}
& \left\langle\downarrow, \sigma_{z}^{-}\right|\left\langle\downarrow, \sigma_{z}^{+}\right|\left\langle\downarrow, X_{1 / 2}\right|\left\langle\downarrow, X_{3 / 2}\right|\left\langle\uparrow, \sigma_{z}^{-}\right|\left\langle\uparrow, \sigma_{z}^{+}\right|\left\langle\uparrow, X_{1 / 2}\right|\left\langle\uparrow, X_{3 / 2}\right|
\end{aligned}
$$

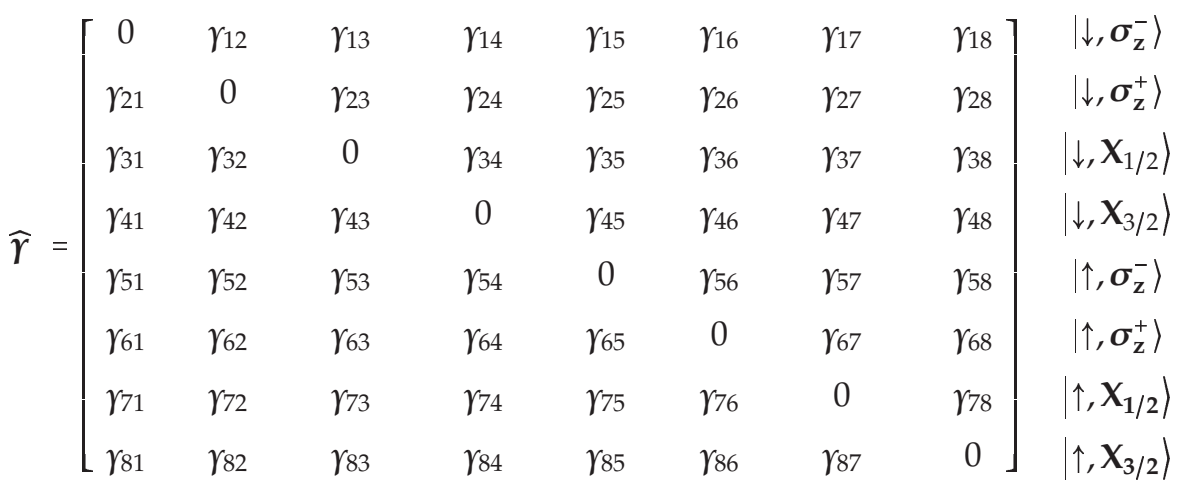

where each element $\gamma_{\mathrm{ij}}$ is calculated according to (3.29). 
Then, the amount of entanglement between the excess electron spin and the single photon polarization can be worked out from the density matrix. The normalized linear entropy is considered

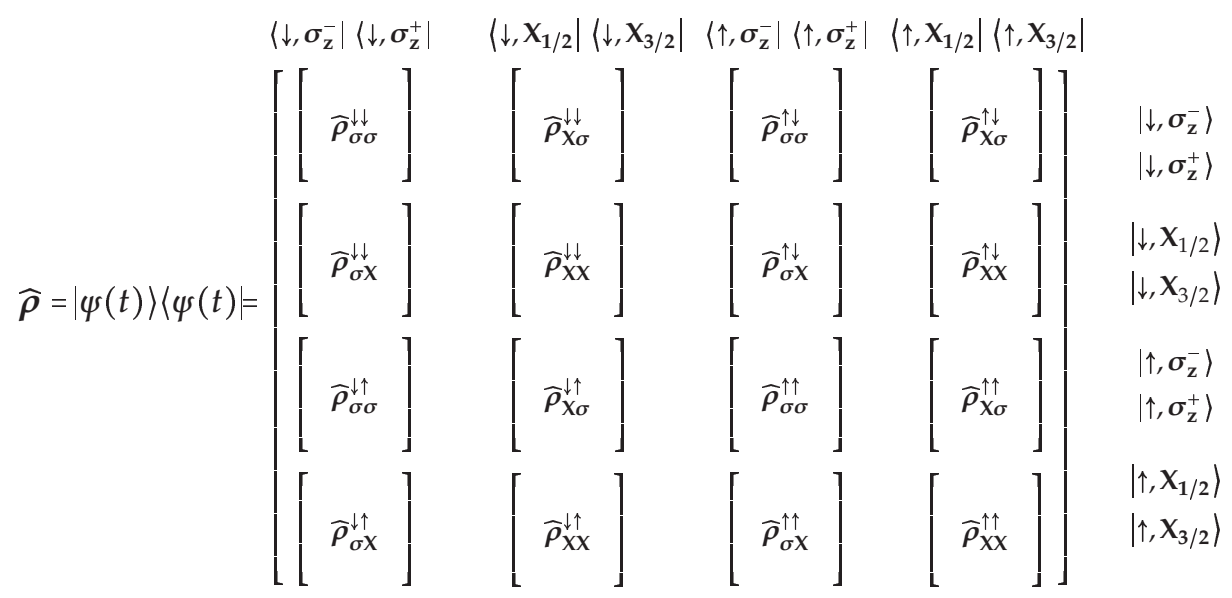

Tracing the density matrix over the cavity polariton results in the spin reduced density matrix such that

$$
\begin{aligned}
& \operatorname{Tr}_{\text {Polariton }} \widehat{\boldsymbol{\rho}}=\widehat{\boldsymbol{\rho}}_{\text {red,spin }} \\
& =\operatorname{Tr}_{\text {Photon }} \widehat{\boldsymbol{\rho}}+\operatorname{Tr}_{\text {Exciton } \boldsymbol{\rho}} \\
& =\left\langle\sigma_{\mathrm{z}}^{+}|\widehat{\rho}| \sigma_{\mathrm{z}}^{+}\right\rangle+\left\langle\sigma_{\mathrm{z}}^{-}|\widehat{\rho}| \sigma_{\mathrm{z}}^{-}\right\rangle+\left\langle\mathrm{X}_{3 / 2}|\widehat{\rho}| \mathbf{X}_{3 / 2}\right\rangle+\left\langle\mathrm{X}_{1 / 2}|\widehat{\rho}| \mathbf{X}_{1 / 2}\right\rangle
\end{aligned}
$$

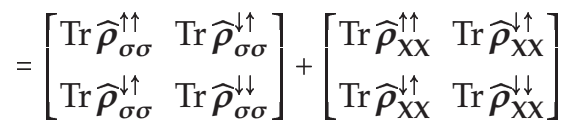

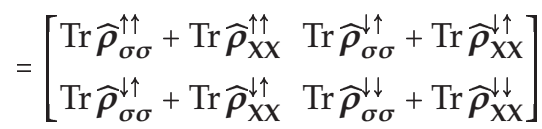

$$
\begin{aligned}
& =2 \times 2 \text {, }
\end{aligned}
$$

where

$$
\begin{aligned}
& \widehat{\rho}_{\sigma \sigma}^{\uparrow \uparrow}=\left[\begin{array}{cc}
\rho_{\sigma_{z}^{+} \sigma_{z}^{+}}^{\uparrow \uparrow} & \rho_{\sigma_{z}^{+} \sigma_{z}^{-}}^{\uparrow \uparrow} \\
\rho_{\sigma_{z}^{-} \sigma_{z}^{+}} & \rho_{\sigma_{z}^{-} \sigma_{z}^{-}}^{\uparrow \uparrow}
\end{array}\right] \widehat{\rho}_{\sigma \sigma}^{\uparrow \downarrow}=\left[\begin{array}{cc}
\rho_{\sigma_{z}^{+} \sigma_{z}^{+}}^{\uparrow \downarrow} & \rho_{\sigma_{z}^{+} \sigma_{z}^{-}}^{\uparrow \downarrow} \\
\rho_{\sigma_{z}^{-} \sigma_{z}^{+}}^{\uparrow} & \rho_{\sigma_{z}^{-} \sigma_{z}^{-}}^{\uparrow}
\end{array}\right], \\
& \widehat{\rho}_{\sigma \sigma}^{\downarrow \uparrow}=\left[\begin{array}{cc}
\rho_{\sigma_{z}^{+} \sigma_{z}^{+}}^{\downarrow \uparrow} & \rho_{\sigma_{z}^{+} \sigma_{\bar{z}}^{-}}^{\downarrow \uparrow} \\
\rho_{\sigma_{z}^{-} \sigma_{z}^{+}}^{\downarrow \uparrow} & \rho_{\sigma_{z}^{-} \sigma_{z}^{-}}^{\downarrow \uparrow}
\end{array}\right] \widehat{\rho}_{\sigma \sigma}^{\downarrow \downarrow}=\left[\begin{array}{cc}
\rho_{\sigma_{z}^{+} \sigma_{z}^{+}}^{\downarrow \downarrow} & \rho_{\sigma_{z}^{+} \sigma_{\bar{z}}^{-}}^{\downarrow} \\
\rho_{\sigma_{z}^{-} \sigma_{z}^{+}}^{\downarrow} & \rho_{\sigma_{z}^{-} \sigma_{z}^{-}}^{\downarrow \downarrow}
\end{array}\right],
\end{aligned}
$$

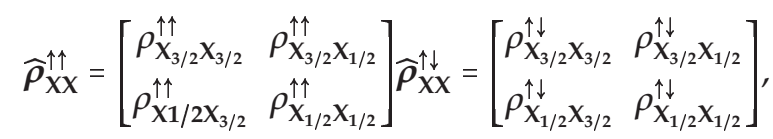

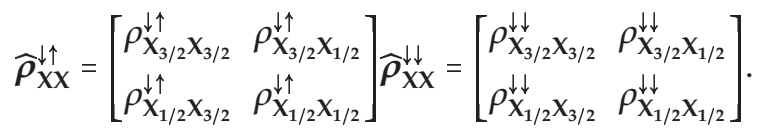




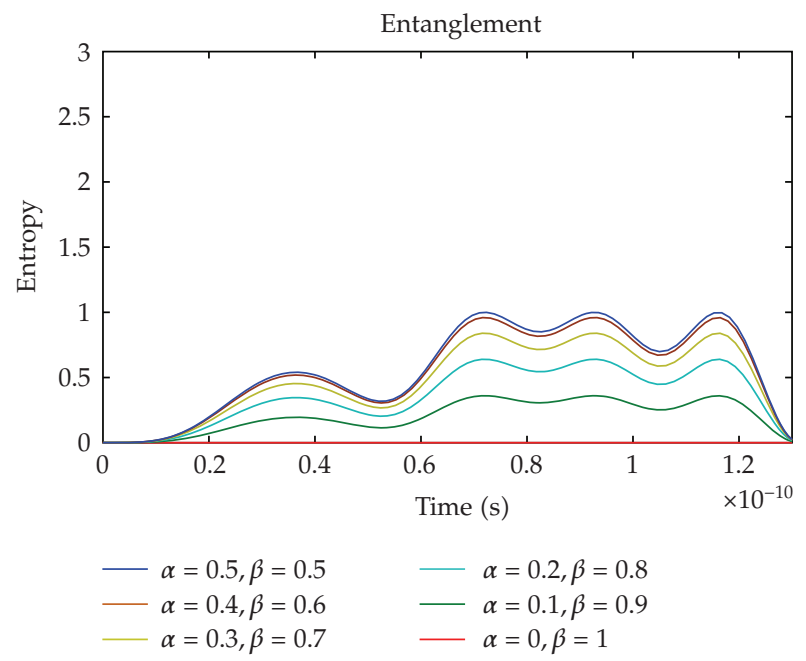

Figure 7: Spin-photon entanglement dynamics for small $\Delta$.

Next, the $2 \times 2$ matrix $\operatorname{Tr}_{\text {Polariton }} \widehat{\rho}$ needs to be squared. As a result, we obtain

$$
\begin{aligned}
{\left[\operatorname{Tr}_{\text {Polariton }} \widehat{\boldsymbol{\rho}}\right]^{2} } & =\left[\begin{array}{ll}
\operatorname{Tr} \widehat{\boldsymbol{\rho}}_{\sigma \sigma}^{\uparrow \uparrow}+\operatorname{Tr} \hat{\boldsymbol{\rho}}_{\mathbf{X X}}^{\uparrow \uparrow} & \operatorname{Tr} \widehat{\boldsymbol{\rho}}_{\sigma \sigma}^{\uparrow \downarrow}+\operatorname{Tr} \widehat{\boldsymbol{\rho}}_{\mathbf{X X}}^{\uparrow \downarrow} \\
\operatorname{Tr} \widehat{\boldsymbol{\rho}}_{\sigma \sigma}^{\downarrow \uparrow}+\operatorname{Tr} \hat{\boldsymbol{\rho}}_{\mathbf{X X}}^{\downarrow \uparrow} & \operatorname{Tr} \widehat{\boldsymbol{\rho}}_{\sigma \sigma}^{\downarrow \downarrow}+\operatorname{Tr} \widehat{\boldsymbol{\rho}}_{\mathbf{X X}}^{\downarrow \downarrow}
\end{array}\right]^{2} \\
& =\left[\begin{array}{ll}
{[\mathcal{A} \mathcal{A}+\mathcal{B O}]} & {[\mathcal{A B}+\mathcal{B Z}]} \\
{[\mathcal{O} \mathcal{A}+\mathcal{Z} \mathcal{O}]} & {[\mathcal{O B}+\mathcal{Z} \mathcal{Z}]}
\end{array}\right]
\end{aligned}
$$

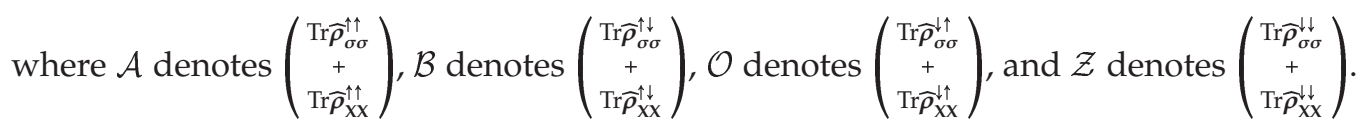

Finally, we take the trace of this square matrix over the spin such that

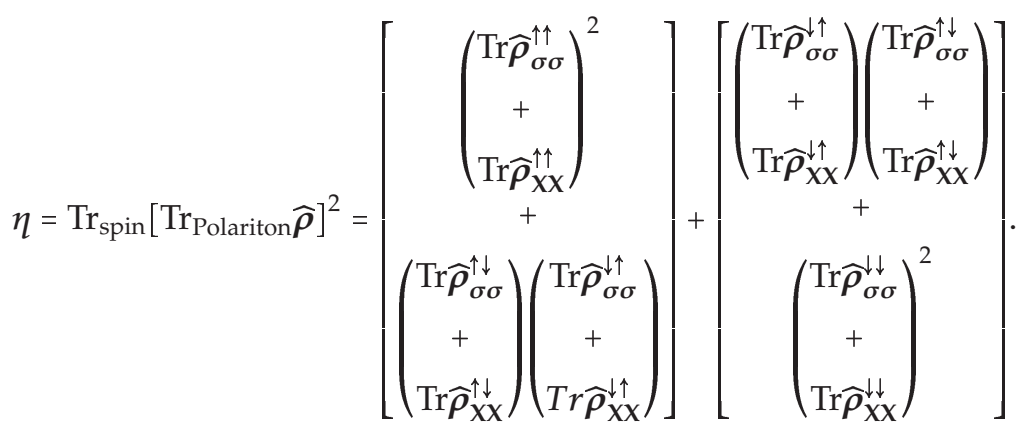

The normalized linear entropy is then

$$
S_{\mathrm{NL}}=2[1-\eta]
$$




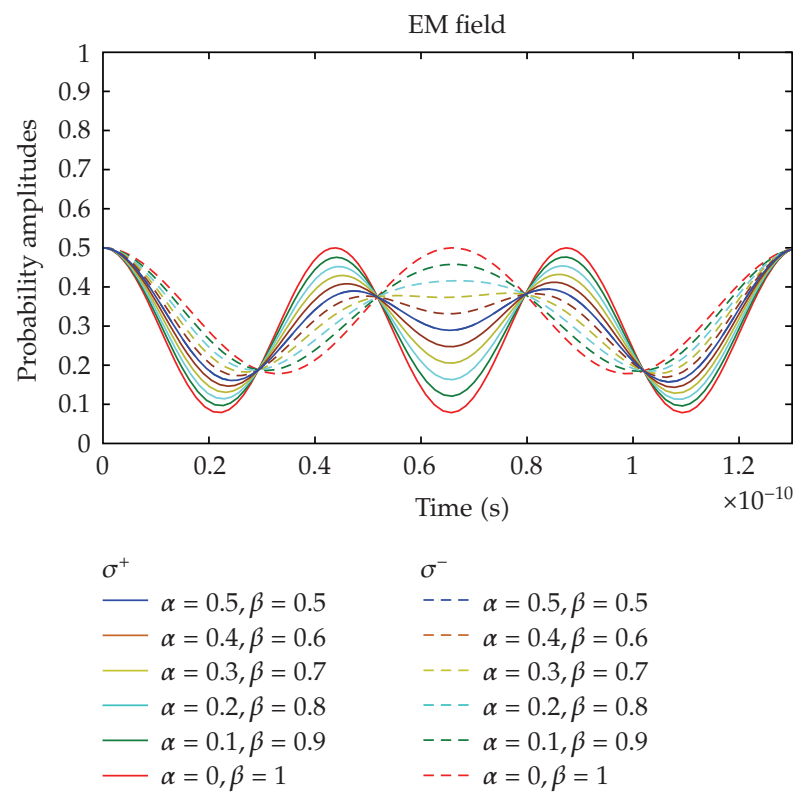

Figure 8: Right and left circular polarization prob. amplitudes for small $\Delta$.

\section{Results}

It is assumed that a GaAs/InGaAs QD with emission wavelength $\lambda_{\mathrm{QD}}$ of $1.182 \mu \mathrm{m}$, which corresponds to the frequency $\omega_{\mathrm{QD}}=1.594 \cdot 10^{15} \mathrm{rad} / \mathrm{s}$, is placed inside a photonic crystal cavity. Next, assuming the dipole moment associated with the InGaAs QD for interactions involving $\left|h_{3 / 2}\right\rangle$ electrons is $\mu_{\mathrm{vc}}=29 \mathrm{D}$, the frequency of the photon $\omega=\omega_{\mathrm{QD}}-\Delta=1.594 \cdot 10^{15} \mathrm{rad} / \mathrm{s}$, and the cavity mode volume $V_{0}=0.039 \mu \mathrm{m}^{3}$, the interaction frequency $g_{3 / 2}$ involving the valence states $\left|h_{3 / 2}\right\rangle$ was found to be $132 \cdot 10^{9} \mathrm{rad} / \mathrm{s}$ leading to the interaction energy in the Hamiltonian $V_{h_{(3 / 2)}}=\hbar g_{3 / 2}$ and the interaction frequency $g_{1 / 2}$ involving the valence states $\left|h_{1 / 2}\right\rangle$ to be $g_{3 / 2} / \sqrt{3}=76 \cdot 10^{9} \mathrm{rad} / \mathrm{s}$ corresponding to the interaction energy $V_{h_{1 / 2}}=\hbar g_{1 / 2}$. First, the entanglement dynamics is considered without decoherence, and then the effects of various decoherence processes on the entanglement dynamics are investigated.

\subsection{Entanglement Dynamics without Decoherence}

There are two regimes of interest when studying the dynamics entanglement inside the cavity with or without decoherence, namely, the small and large energy detuning regimes with respect to the QD and the field. In both regimes, we consider interaction times needed to rotate the linear polarization of the photon by 90 degrees from one pure state to the other expecting the condition of maximum entanglement to occur somewhere in the range during which the photon is in a superposition of its polarization eigenstates.

\subsubsection{Case 1: Small $\Delta$, Spin Initialized to $\alpha|\downarrow\rangle+\beta|\uparrow\rangle$, Photon Initialized to $|\uparrow\rangle$}

The "small" detuning energy is selected to be $\Delta=78 \mu \mathrm{eV}$, which corresponds to the optimized value $\Delta=0.86 g_{3 / 2}$ so as to have a photon and not an exciton back into the cavity once the 
polarization has rotated by 90 degrees or $2 \varphi=\pi$. This takes approximately 130 picoseconds. The density matrix is initialized to (4.1), where $\alpha$ and $\beta$ are the probability amplitudes associated with spin state $|\downarrow\rangle$ and spin state $|\uparrow\rangle$, respectively,

$$
\widehat{\rho}(\mathrm{t}=0)=\frac{1}{2} \cdot\left[\begin{array}{ccccccccc}
\left\langle\downarrow, \sigma_{\mathbf{z}}^{+}\right| & \left\langle\downarrow, \sigma_{\mathbf{z}}^{-}\right| & \left\langle\downarrow, \mathbf{X}_{1 / 2}\right| & \left\langle\downarrow, \mathbf{X}_{3 / 2}\right| & \left\langle\uparrow, \sigma_{\mathbf{z}}^{-}\right| & \left\langle\uparrow, \sigma_{\mathbf{z}}^{+}\right| & \left\langle\uparrow, \mathbf{X}_{1 / 2}\right| & \left\langle\uparrow, \mathbf{X}_{3 / 2}\right| & \\
|\alpha|^{2} & |\alpha|^{2} & 0 & 0 & \alpha^{*} \beta & \alpha^{*} \beta & 0 & 0 \\
0 & |\alpha|^{2} & 0 & 0 & \alpha^{*} \beta & \alpha^{*} \beta & 0 & 0 \\
0 & 0 & 0 & 0 & 0 & 0 & 0 & 0 \\
\beta^{*} \alpha & \beta^{*} \alpha & 0 & 0 & |\beta|^{2} & |\beta|^{2} & 0 & 0 \\
\beta^{*} \alpha & \beta^{*} \alpha & 0 & 0 & |\beta|^{2} & |\beta|^{2} & 0 & 0 \\
0 & 0 & 0 & 0 & 0 & 0 & 0 & 0 \\
0 & 0 & 0 & 0 & 0 & 0 & 0 & 0
\end{array}\right] \begin{gathered}
\left|\downarrow, \sigma_{\mathbf{z}}^{-}\right\rangle \\
\left|\downarrow, \mathbf{X}_{1 / 2}^{+}\right\rangle \\
\left|\downarrow, \mathbf{X}_{3 / 2}\right\rangle \\
\left|\uparrow, \sigma_{\mathbf{z}}^{-}\right\rangle \\
\left|\uparrow, \sigma_{\mathbf{z}}^{+}\right\rangle \\
\left|\uparrow, \mathbf{X}_{1 / 2}\right\rangle \\
\left|\uparrow, \mathbf{X}_{3 / 2}\right\rangle
\end{gathered}
$$

The results are shown in Figures 5, 6, 7, and 8 . In the small $\Delta$ regime, there are three peaks or maxima in the calculated electron-photon system entropy, which means that there are three different conditions for maximum entanglement. This differs somehow from the prediction obtained from the standard parameterization approach [14]. These peaks occur after 71,93, and 116 picoseconds of interaction time. However, there is a trade-off since the probability of creating an exciton is nearly 0.4 or $40 \%$ for the first 2 peaks and $45 \%$ for the last peak. This is not desirable from an engineering point of view since the aim would be to have a high probability of releasing the photon back into the quantum network waveguides once maximally entangled. Entanglement is also confirmed by the fact that the Poincare sphere shows intermediate polarization states inside the unit sphere .

\subsubsection{Case 2: Large $\Delta$, Spin Initialized to $\alpha|\downarrow\rangle+\beta|\uparrow\rangle$, Photon Initialized to $|\uparrow\rangle$}

Here, the "large" detuning energy is selected to be $\Delta=1.5 \mathrm{meV}$, and the 90 degrees rotation of the linear polarization takes place in approximately 1.225 nanoseconds. The results are shown in Figures $9,10,11$. and 12 . In the large $\Delta$ regime, there is only one peak or maxima of magnitude 1 in the calculated electron-photon system entropy, as predicted in by the standard parameterization approach [14]. This peak occurs at 0.625 nanoseconds for all cases. Furthermore, the probability of creating an exciton is effectively always zero. This is great from an engineering point of view since there will always be a high probability of releasing a photon back into the quantum network waveguides once maximally entangled.

\subsection{Entanglement Dynamics with Decoherence}

Recently, the hyperfine coupling with the nuclear spins of the semiconductor host material $\left(\sim 10^{5}\right.$ nuclei in a single quantum dot), which acts as a random magnetic field (up to a few 


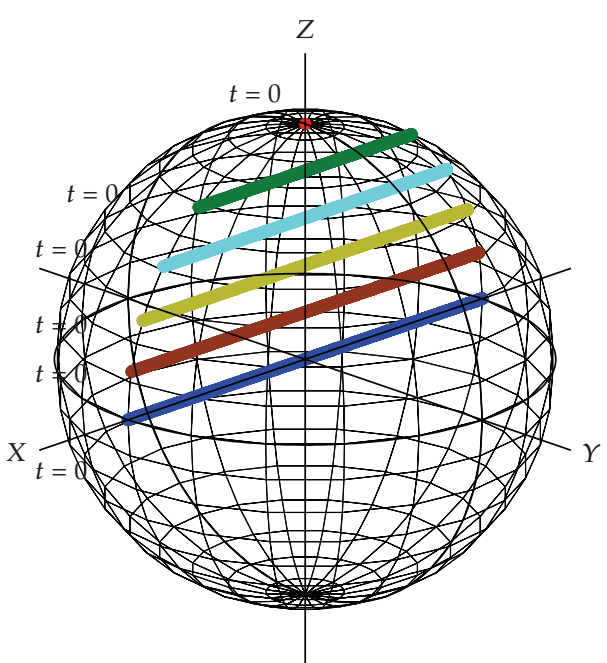

(a)
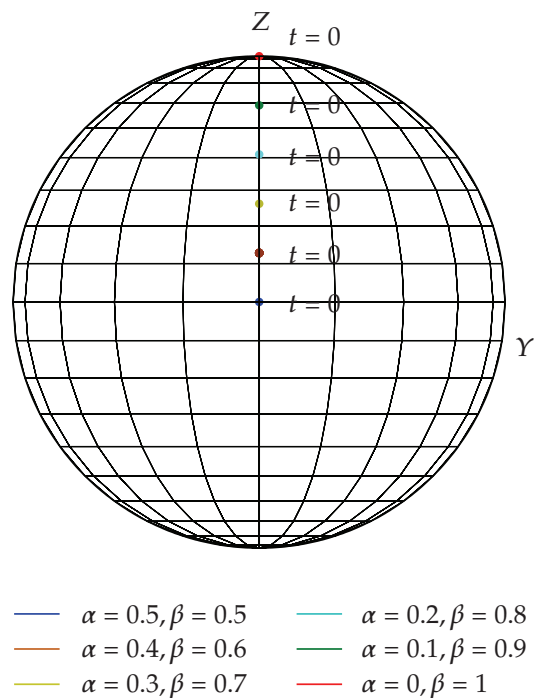

(c)

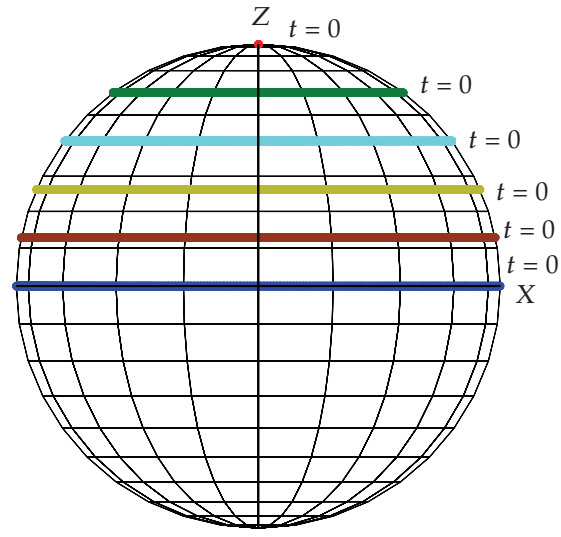

(b)

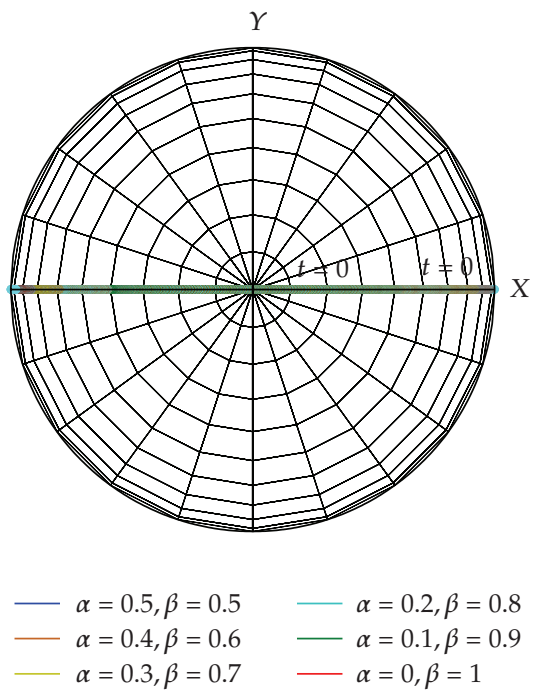

(d)

Figure 9: Excess electron spin dynamics on the bloch sphere for large $\Delta$.

Tesla in InAs quantum dots), has been identified as the ultimate limit, at low temperature, to the electron spin relaxation or decoherence in quantum dots. Consequently, assuming low temperature conditions, only the various decoherence processes associated with hyperfine interactions will be considered; where as, those associated with phonon interactions are ignored.

First, the Fermi contact term is considered. It relates to the direct interaction of the nuclear dipole with the spin dipoles and is only non-zero for states with a finite electron spin density at the position of the nucleus (those with unpaired electrons in the conduction band). Therefore, the Fermi contact hyperfine interaction does not affect the trion states, just the 


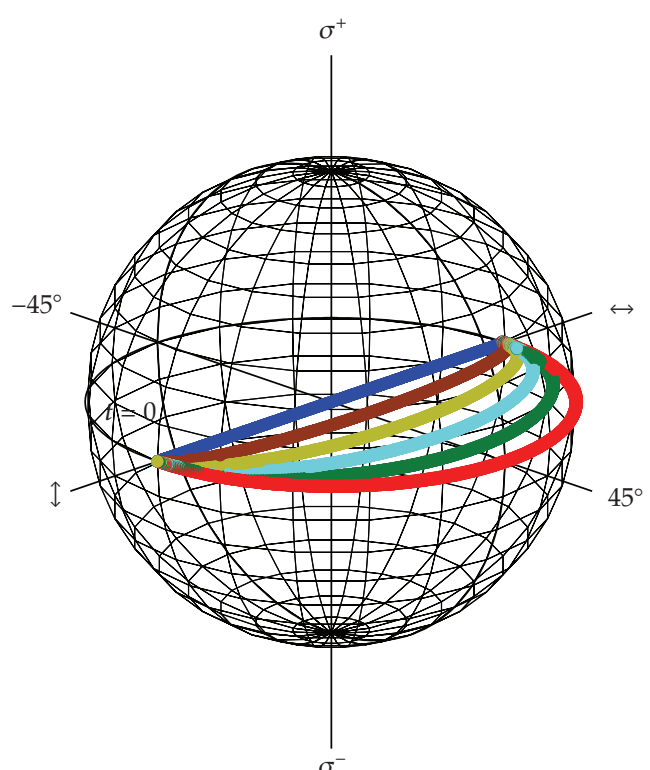

(a)
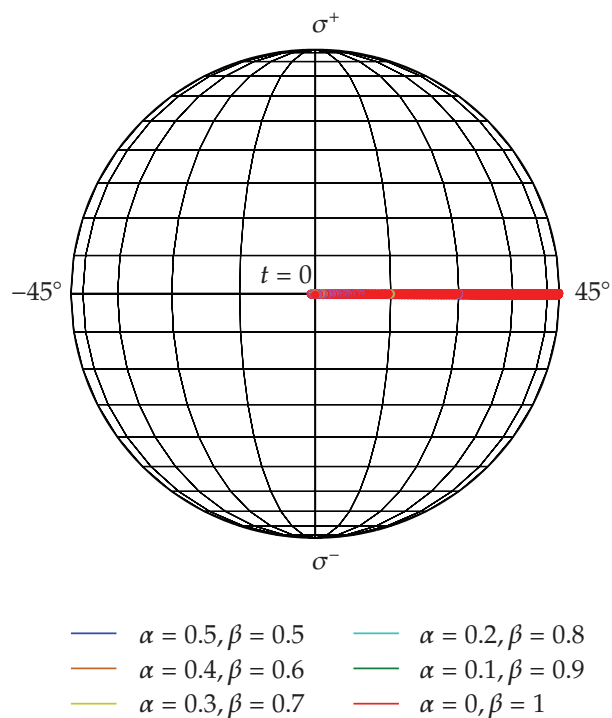

(c)

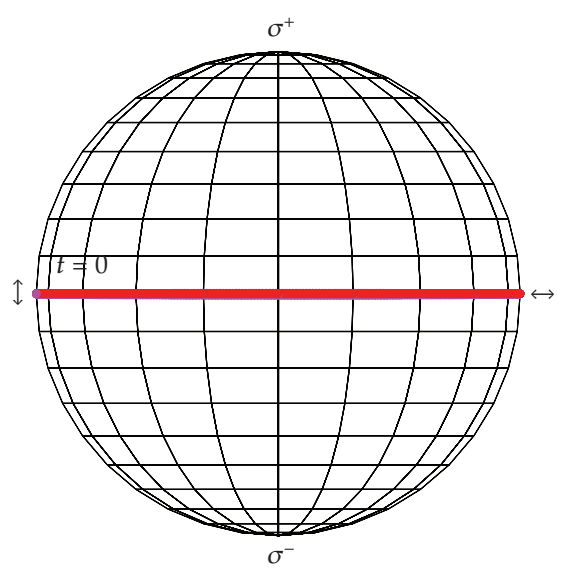

(b)

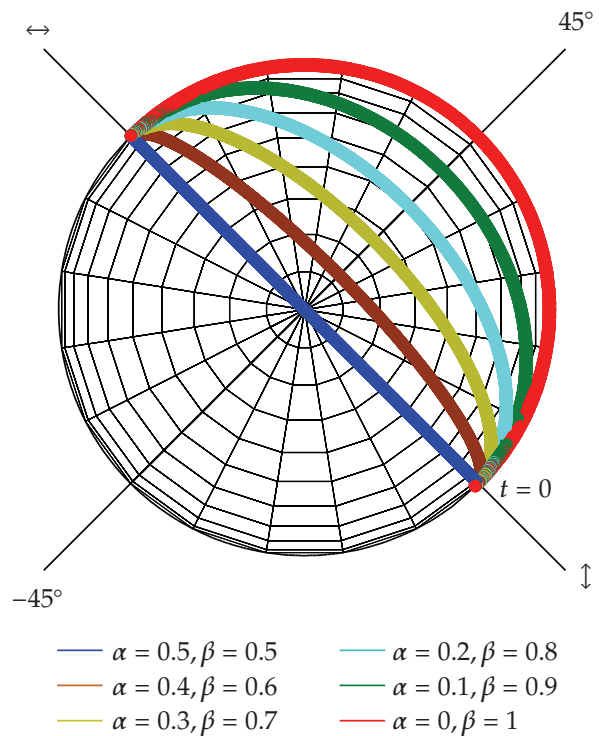

(d)

Figure 10: Photon polarization dynamics on the poincare sphere for large $\Delta$.

following states $\left|\downarrow, \sigma_{\mathrm{z}}^{-}\right\rangle,\left|\downarrow, \sigma_{\mathrm{z}}^{+}\right\rangle,\left|\uparrow, \sigma_{\mathrm{z}}^{-}\right\rangle$, and $\left|\uparrow, \sigma_{\mathrm{z}}^{+}\right\rangle$. A typical longitudinal relaxation time for semiconductors is on the order of $T_{1} \simeq 1 \mathrm{~ns}$ [15].

Second, due to the $p$ symmetry of the Bloch wavefunctions in the valence band, the coupling of the nuclei with holes can be generally neglected because the Fermi contact interaction vanishes. The coupling constants of the hole-nuclear interaction are significantly smaller than the coupling constants of the electron-nuclear interaction. One recent reference work determined the longitudinal relaxation time for hole-nuclear interaction to be 


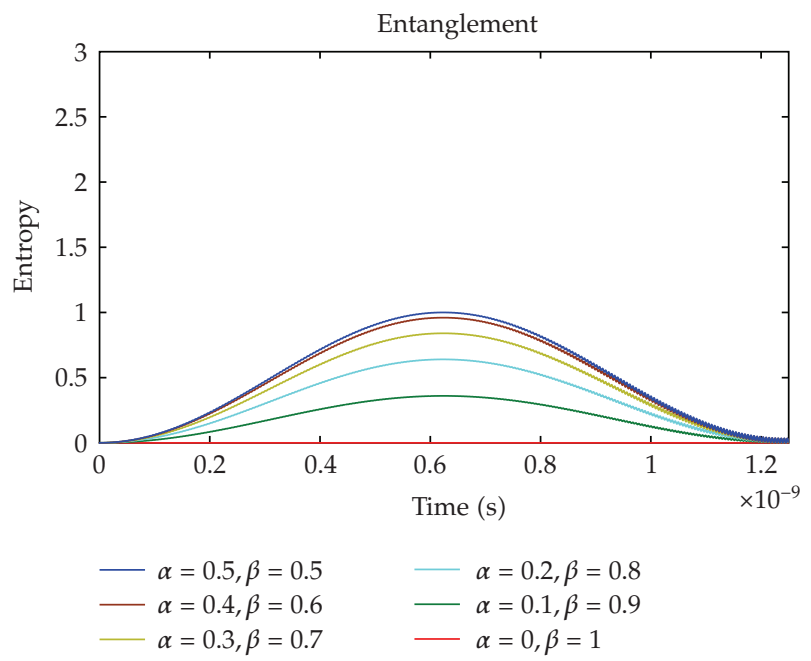

Figure 11: Spin-photon entanglement dynamics for large $\Delta$.

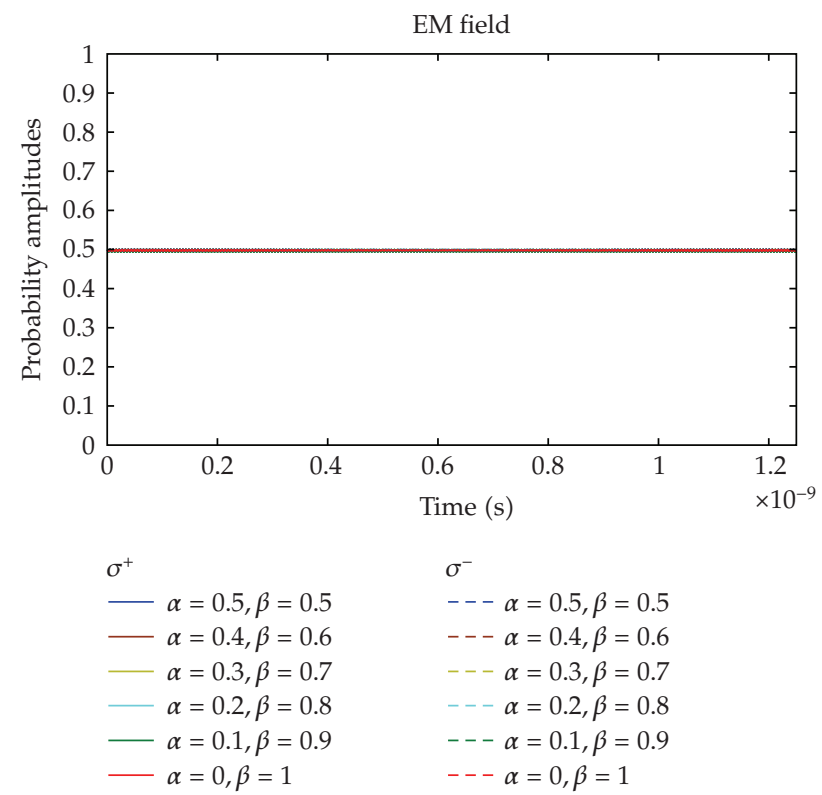

Figure 12: Right and left circular polarization prob. amplitudes for large $\Delta$.

$T_{1}=14 \mathrm{~ns}[16]$. Furthermore, all trion states are affected, namely, $\left|\downarrow, \mathbf{X}_{1 / 2}\right\rangle,\left|\downarrow, \mathbf{X}_{3 / 2}\right\rangle,\left|\uparrow, \mathbf{X}_{1 / 2}\right\rangle$, and $\left|\uparrow, \mathbf{X}_{3 / 2}\right\rangle$. It is assumed that $T_{1}$ is the same for both $\left|h_{1 / 2}\right\rangle$-nucleus and $\left|h_{3 / 2}\right\rangle$-nucleus interactions. It is also interesting to note that there are not any dark states for trions.

Last, radiative recombination rates of trions are also included in our model of decoherence. In the case of InGaAs quantum dots with an emission wavelength of $\lambda=950 \mathrm{~nm}$, a radiative recombination lifetime of $\tau_{X^{-}}=0.84 \mathrm{~ns}$ for negatively charged excitons was reported [17] as well as $\tau_{X^{-}}=1.24 \mathrm{~ns}$ in the case of InAs quantum dots with an emission wavelength of $\lambda=850 \mathrm{~nm}$ [18]. On the other hand, GaAs quantum dots spin relaxation as 


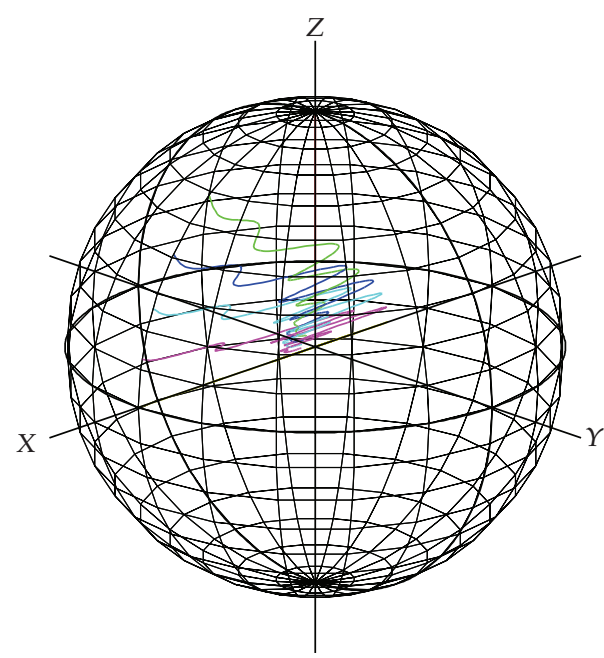

(a)
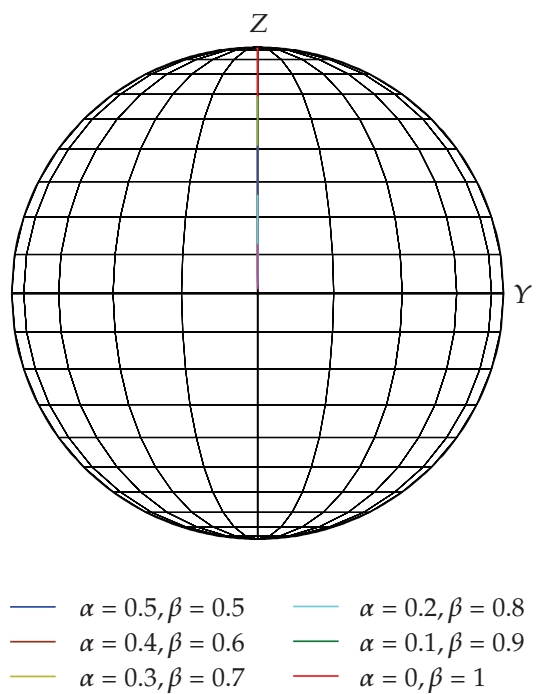

(c)

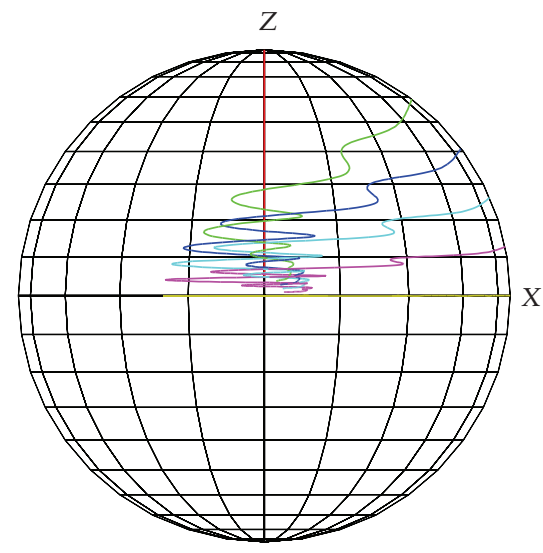

(b)

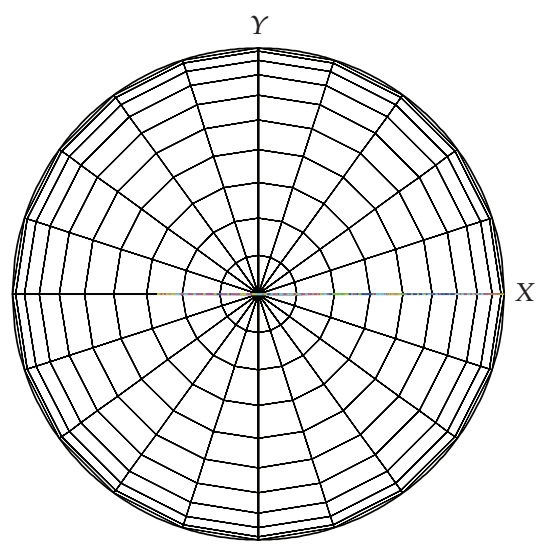

$$
\begin{aligned}
\alpha & =0.5, \beta=0.5 \quad \square \\
\alpha & =0.4, \beta=0.6 \\
-\alpha & =0.2, \beta=0.8 \\
\alpha & =0.3, \beta=0.7 \quad-\alpha=0.1, \beta=0.9 \\
& \alpha=0, \beta=1
\end{aligned}
$$

(d)

Figure 13: Excess electron spin dynamics on the bloch sphere for small $\Delta$.

phonon-assisted Dresselhaus spin-orbit scattering was estimated $T_{1}=34 \mu \mathrm{s}$ at $T=4.5 \mathrm{~K}$. This illustrates the fact that these types of decoherence processes can be ignored at low temperature.

\subsubsection{Case 1: Small $\Delta$, Spin Initialized to $\alpha|\downarrow\rangle+\beta|\uparrow\rangle$, Photon Initialized to $|\uparrow\rangle$}

The "small" detuning energy is again selected to be $\Delta=78 \mu \mathrm{eV}$. The results are shown in figures $13,14,15$, and 16 . In the small $\Delta$ regime, for all case of the excess electron spin being in a superposition of its eigenstates, a decent amount of entanglement ( $35 \%$ entanglement) is 


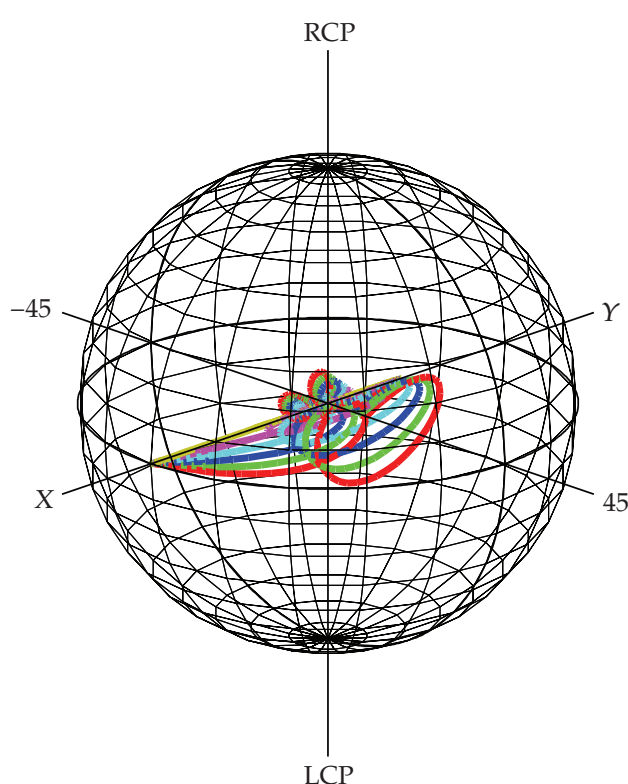

(a)

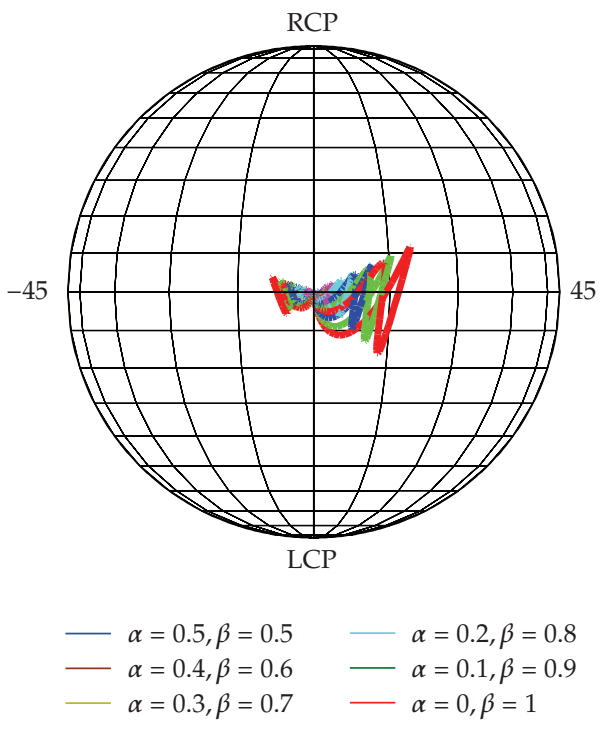

(c)

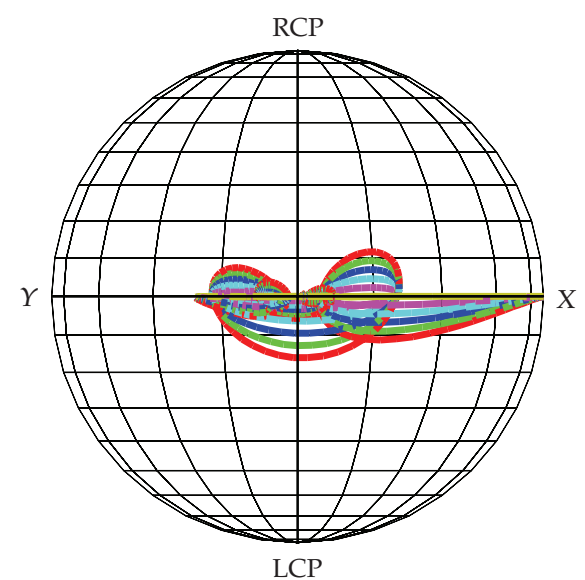

(b)

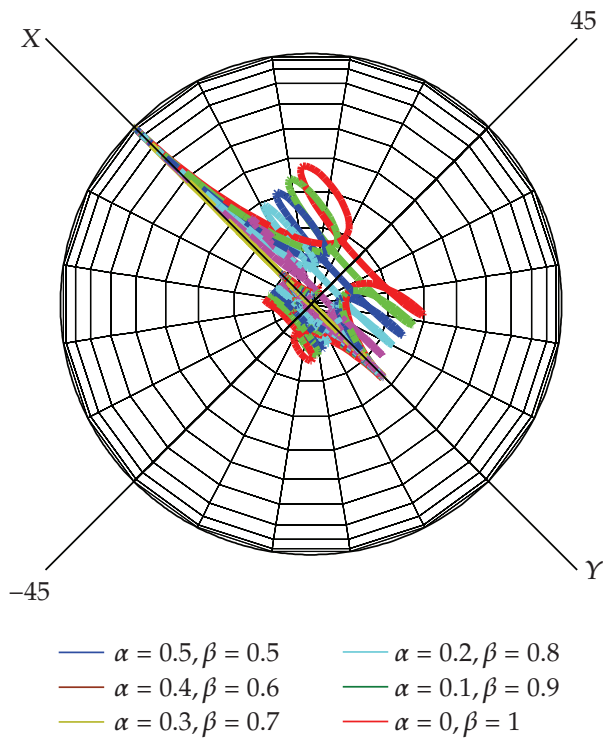

(d)

Figure 14: Photon polarization dynamics on the poincare sphere for small $\Delta$.

obtained within only the first 200 picoseconds. Afterward, all entanglement is lost. Moreover, the dynamics of entanglement is interesting in that for the lower amounts of superposition in the excess spin state, maximum entanglement is reached faster, yet it decreases also faster.

\subsubsection{Case 2: Large $\Delta$, Spin Initialized to $\alpha|\downarrow\rangle+\beta|\uparrow\rangle$, Photon Initialized to $|\uparrow\rangle$}

Also, the "large" detuning energy is selected to be $\Delta=1.5 \mathrm{meV}$. A smaller amount of entanglement is realized, namely, $25 \%$. And, it lasts only within the first 300 picoseconds. 


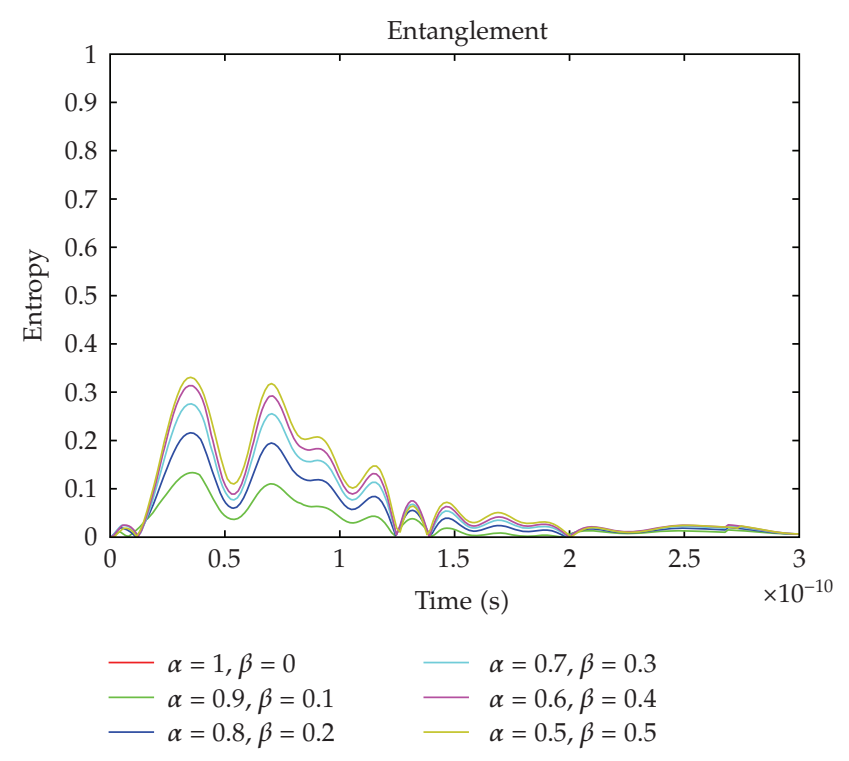

Figure 15: Spin-photon entanglement dynamics for small $\Delta$.

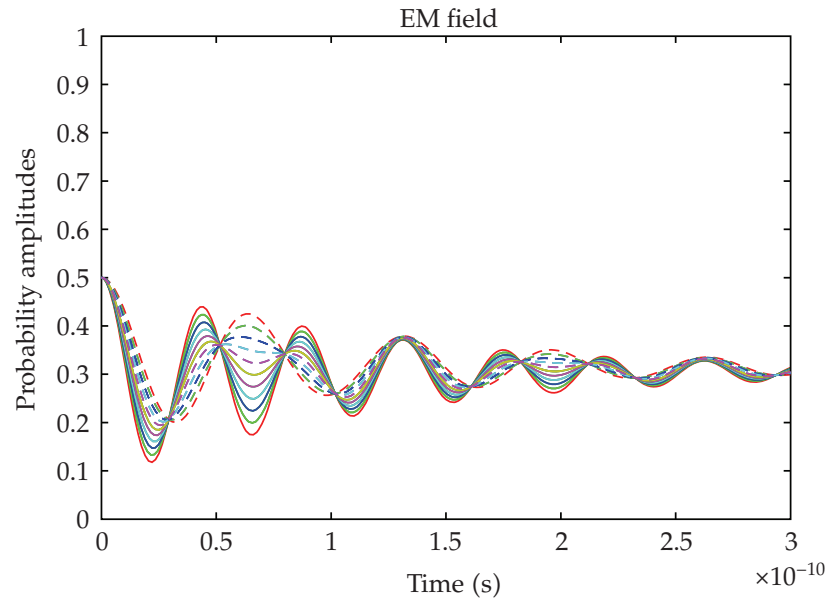

Figure 16: Right and left circular polarization prob. amplitudes for small $\Delta$.

These results are shown in Figures 17, 18, 19, and 20. Here again the dynamics of entanglement is unexpected as lower amounts of superposition in the excess electron spin states result in a larger maximum for the entanglement.

\section{Conclusion}

Some entanglement between an electron spin qubit within a quantum dot and a singlephoton qubit interacting inside a high- $Q$ nanocavity can be obtained even in the presence 


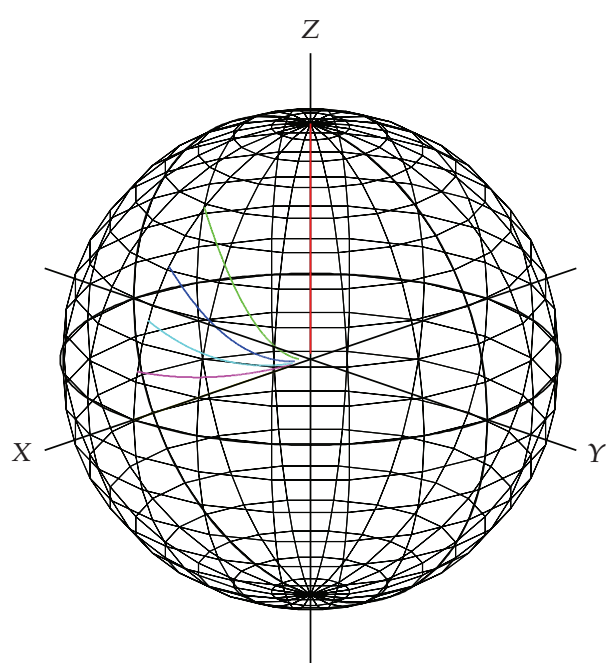

(a)
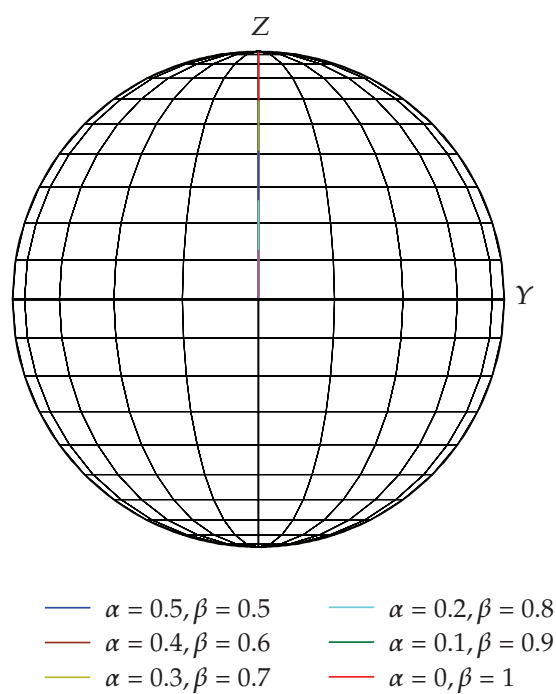

(c)

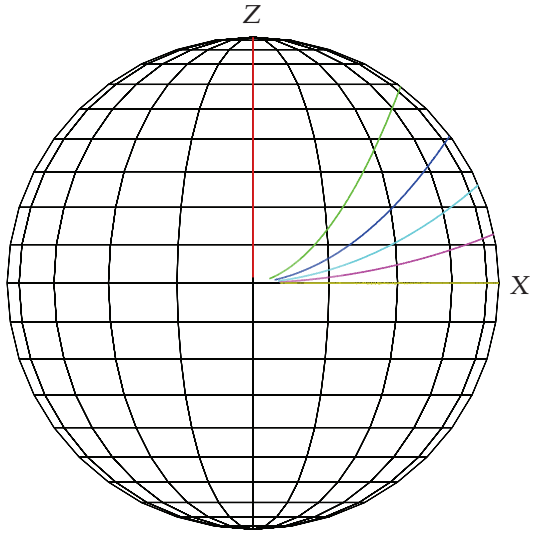

(b)

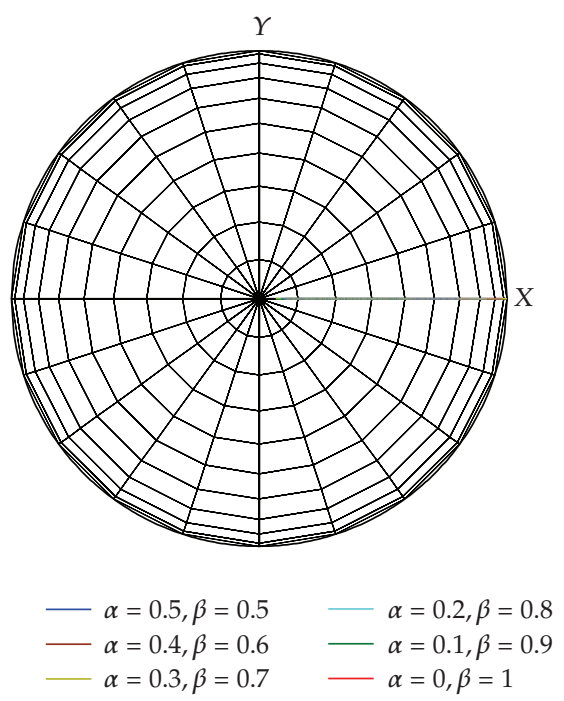

(d)

Figure 17: Excess electron spin dynamics on the bloch sphere for large $\Delta$.

of severe decoherence processes. Whether or not such amount of entanglement for such short periods of time can be useful in a real physical system remains to be seen. For a certainty, the performance of such scheme will have to be improved. There are three areas one could look into.

First, performances could be improved by increasing interaction frequencies. This is done by making it a resonant process, or by reducing the volume of the electromagnetic mode, or by changing material system so as to obtain a strong dipole moment for the quantum dot or by increasing dramatically the quality factor of the cavity. 


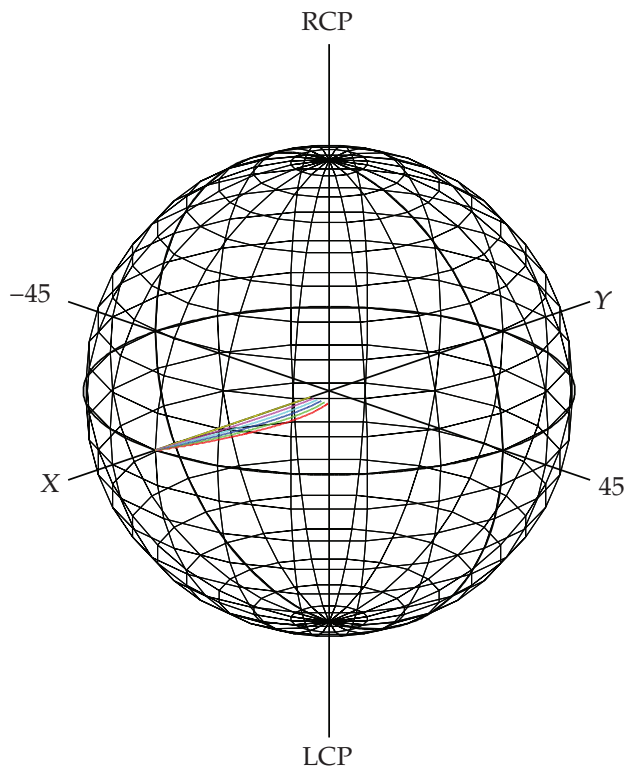

(a)

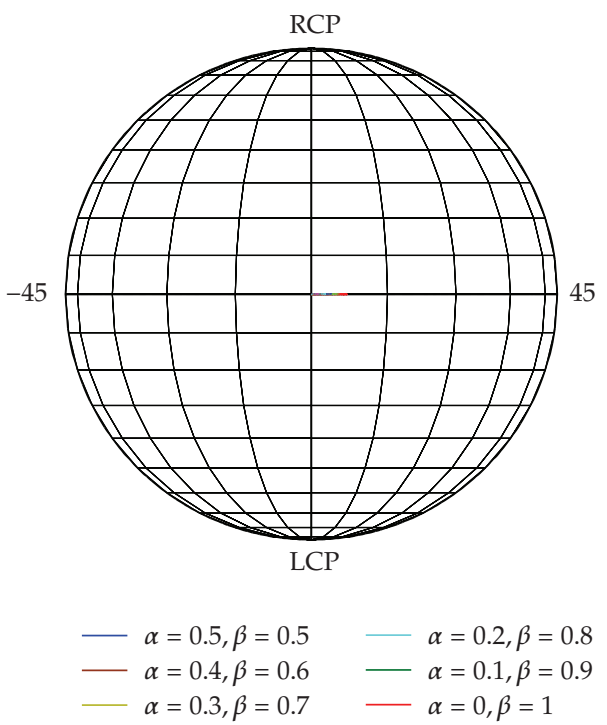

(c)

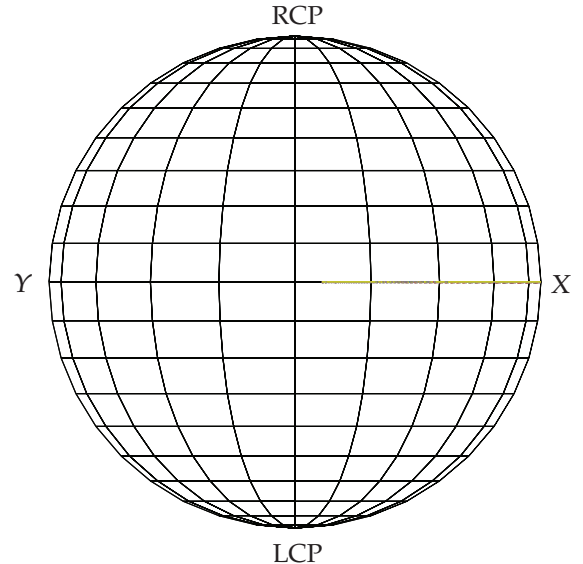

(b)

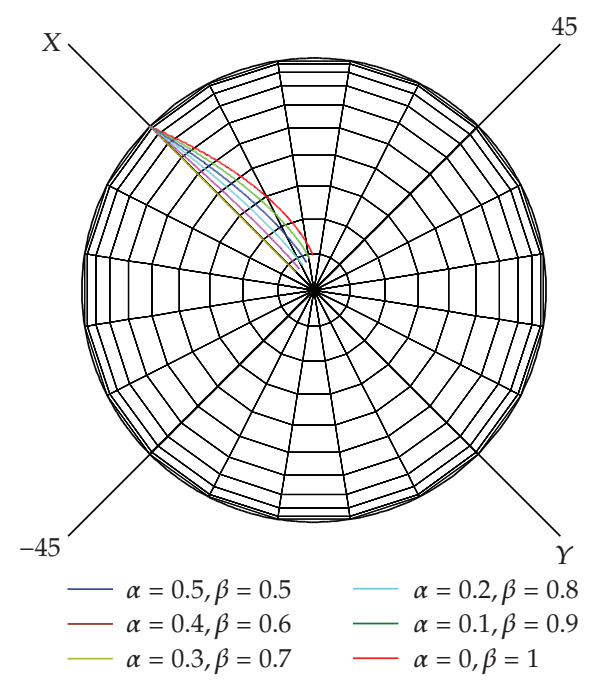

(d)

Figure 18: Photon polarization dynamics on the poincare sphere for large $\Delta$.

Second, performances could be increased by eliminating or reducing the effect of decoherence. Indium atoms has I9/2 spin and arsenic atom have I3/2 spin; which make InAs quantum dots bad candidate as far as decoherence is concern. Other semiconductors with lower or without nucleus spin could be used. The dephasing times of the II-VI compounds are 3-10 times larger than dephasing times for III-V compounds [19]; however, for wurzitetype semiconductors to have similar optical selection rules as in the case of zinc-blende-type semiconductors, propagation along the $c$ axis is needed [20]. 


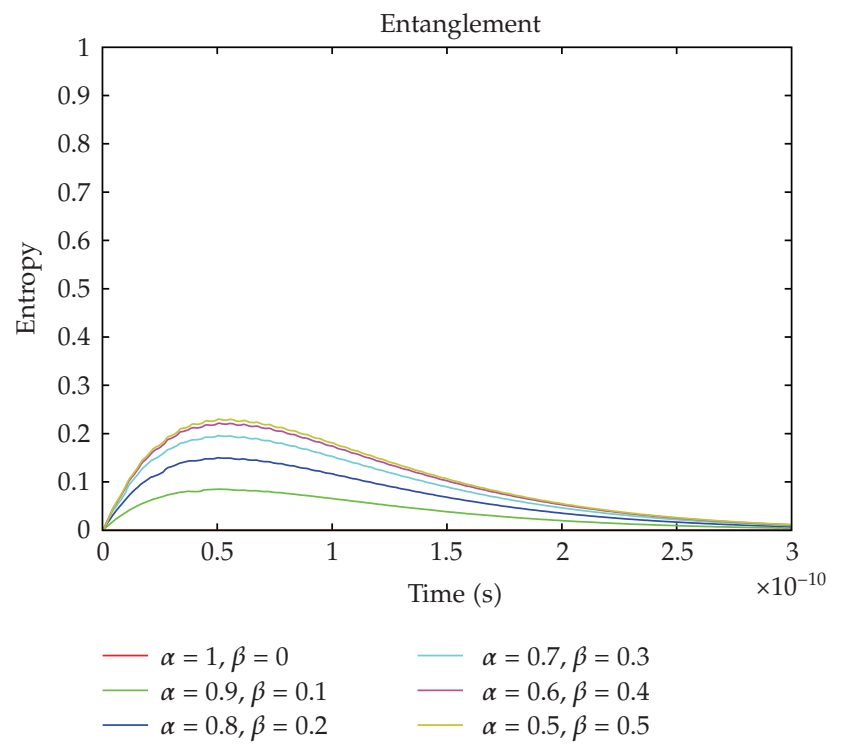

Figure 19: Spin-photon entanglement dynamics for large $\Delta$.

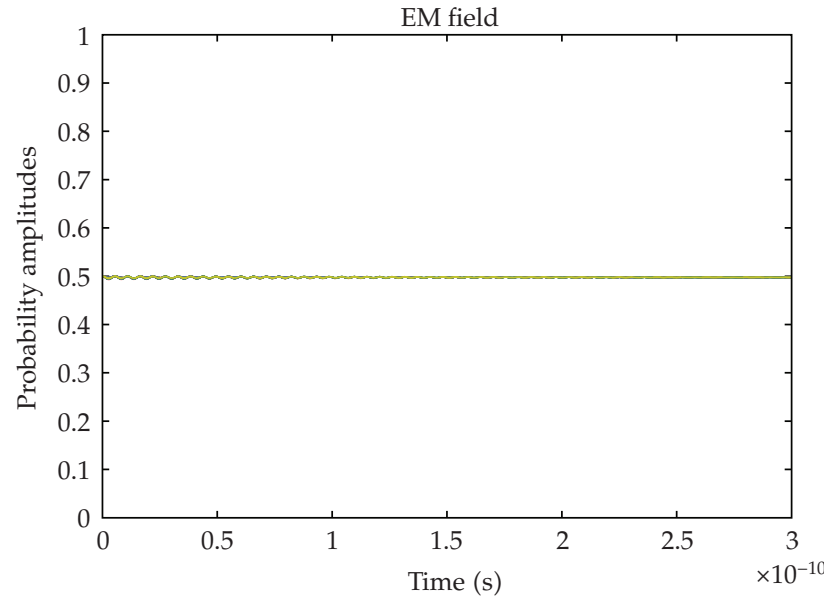

Figure 20: Right and left circular polarization prob. amplitudes for large $\Delta$.

At last, improvements in performances could also be obtained performing manipulations on the nuclear system [21], or using hole spin in the valence band instead of the electron spin in the conduction band as storage qubit since it is less influenced by the nucleus spin.

\section{Acknowledgment}

The authors acknowledge the support from NSF ECCS-0725514. 


\section{References}

[1] D. L. Moehring, M. J. Madsen, K. C. Younge, et al., "Quantum networking with photons and trapped atoms (invited)," Journal of the Optical Society of America B, vol. 24, no. 2, pp. 300-315, 2007.

[2] T. Wilk, S. C. Webster, A. Kuhn, and G. Rempe, "Single-atom single-photon quantum interface," Science, vol. 317, no. 5837, pp. 488-490, 2007.

[3] M. N. Leuenberger, M. E. Flatte, and D. D. Awschalom, “Teleportation of electronic many-qubit states encoded in the electron spin of quantum dots via single photons," Physical Review Letters, vol. 94, no. 10, Article ID 107401, 4 pages, 2005.

[4] D. Englund, A. Faraon, B. Zhang, Y. Yamamoto, and J. Vučković, "Generation and transfer of single photons on a photonic crystal chip," Optics Express, vol. 15, no. 9, pp. 5550-5558, 2007.

[5] M. Kroutvar, Y. Ducommun, D. Heiss, et al., "Optically programmable electron spin memory using semiconductor quantum dots," Nature, vol. 432, no. 7013, pp. 81-84, 2004.

[6] Al. L. Efros, M. Rosen, M. Kuno, M. Nirmal, D. J. Norris, and M. Bawendi, "Band-edge exciton in quantum dots of semiconductors with a degenerate valence band: dark and bright exciton states," Physical Review B, vol. 54, no. 7, pp. 4843-4856, 1996.

[7] A. I. Ekimov, F. Hache, M. C. Schanneklein, et al., "Absorption and intensity-dependent photoluminescence measurements on Cdse quantum dots-assignment of the 1st electronic-transitions," Journal of the Optical Society of America B, vol. 10, pp. 100-107, 1993.

[8] C. Y. Hu, A. Young, J. L. O’Brien, W. J. Munro, and J. G. Rarity, “Giant optical Faraday rotation induced by a single-electron spin in a quantum dot: applications to entangling remote spins via a single photon," Physical Review B, vol. 78, no. 8, Article ID 085307, 5 pages, 2008.

[9] H. P. Seigneur, M. N. Leuenberger, and W. V. Schoenfeld, "Single-photon Mach-Zehnder interferometer for quantum networks based on the single-photon Faraday effect," Journal of Applied Physics, vol. 104, no. 1, Article ID 014307, 2008.

[10] U. Fano, "Description of states in quantum mechanics by density matrix and operator techniques," Reviews of Modern Physics, vol. 29, pp. 74-93, 1957.

[11] M. O. Scully and M. S. Zubairy, Quantum Optics, Cambridge University Press, New York, NY, USA, 1997.

[12] C. H. Bennett, D. P. DiVincenzo, J. A. Smolin, and W. K. Wootters, "Mixed-state entanglement and quantum error correction," Physical Review A, vol. 54, no. 5, pp. 3824-3851, 1996.

[13] A. Silberfarb and I. H. Deutsch, "Entanglement generated between a single atom and a laser pulse," Physical Review A, vol. 69, no. 4, Article ID 042308, 8 pages, 2004.

[14] M. N. Leuenberger, "Fault-tolerant quantum computing with coded spins using the conditional Faraday rotation in quantum dots," Physical Review B, vol. 73, no. 7, Article ID 075312, 8 pages, 2006.

[15] I. A. Merkulov, Al. L. Efros, and M. Rosen, "Electron spin relaxation by nuclei in semiconductor quantum dots," Physical Review B, vol. 65, no. 20, Article ID 205309, 8 pages, 2002.

[16] B. Eble, C. Testelin, P. Desfonds, et al., "Hole-nuclear spin interaction in quantum dots," Physical Review Letters, vol. 102, no. 14, Article ID 146601, 4 pages, 2009.

[17] P. A. Dalgarno, J. M. Smith, J. McFarlane, et al., "Coulomb interactions in single charged selfassembled quantum dots: radiative lifetime and recombination energy," Physical Review B, vol. 77, no. 24, Article ID 245311, 8 pages, 2008.

[18] G. Munoz-Matutano, J. Gomis, B. Alen, et al., "Exciton, biexciton and trion recombination dynamics in a single quantum dot under selective optical pumping," Physica E, vol. 40, no. 6, pp. 2100-2103, 2008.

[19] C. Testelin, F. Bernardot, B. Eble, and M. Chamarro, "Hole-spin dephasing time associated with hyperfine interaction in quantum dots," Physical Review B, vol. 79, no. 19, Article ID 195440, 13 pages, 2009.

[20] G. D. Scholes, "Selection rules for probing biexcitons and electron spin transitions in isotropic quantum dot ensembles," Journal of Chemical Physics, vol. 121, no. 20, pp. 10104-10110, 2004.

[21] B. Eble, P.-F. Braun, O. Krebs, et al., "Spin dynamics and hyperfine interaction in InAs semiconductor quantum dots," Physica Status Solidi B, vol. 243, no. 10, pp. 2266-2273, 2006. 


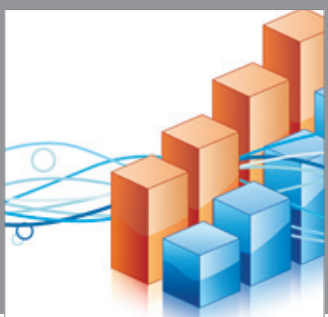

Advances in

Operations Research

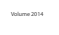

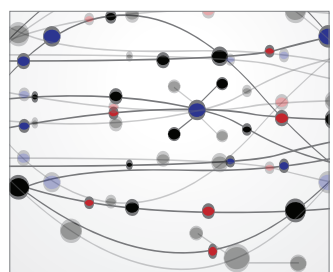

\section{The Scientific} World Journal
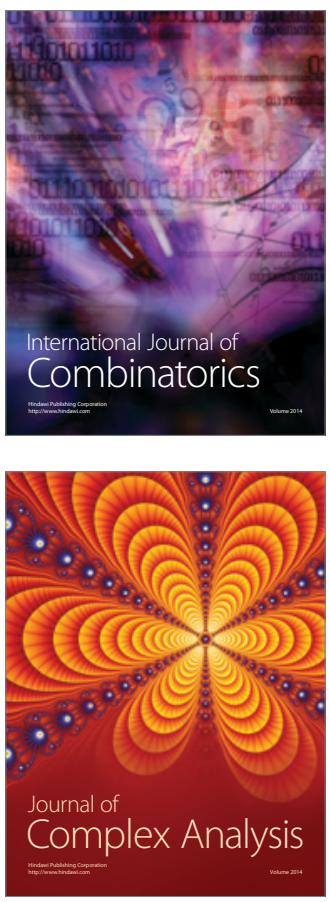

International Journal of

Mathematics and

Mathematical

Sciences
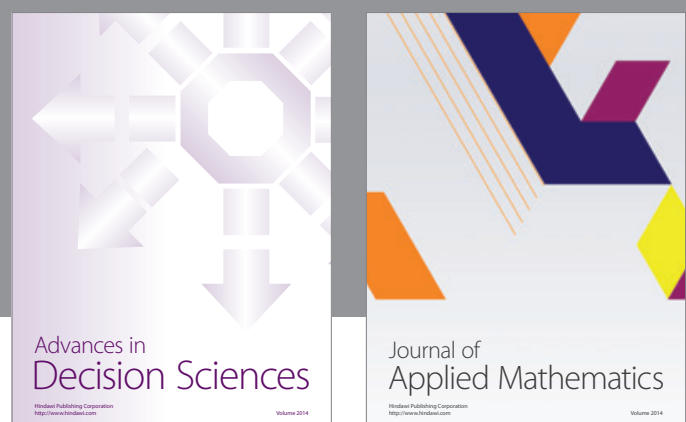

Journal of

Applied Mathematics
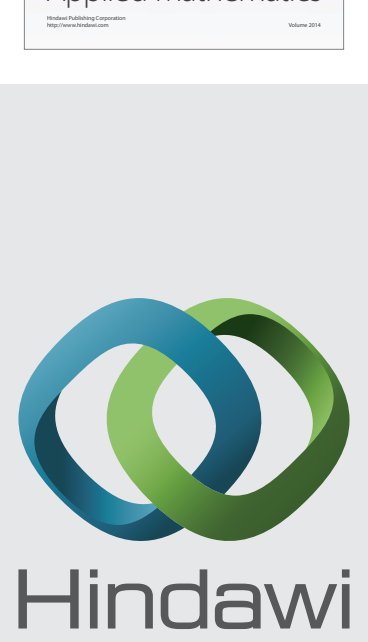

Submit your manuscripts at http://www.hindawi.com
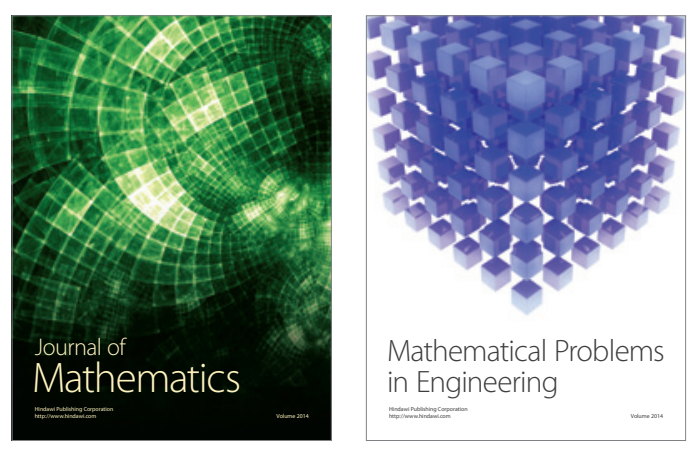

Mathematical Problems in Engineering
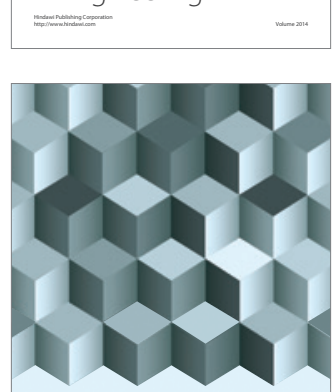

Journal of

Function Spaces
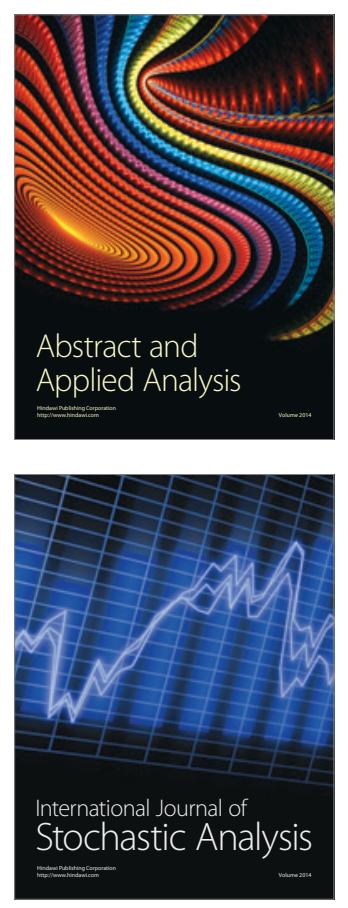

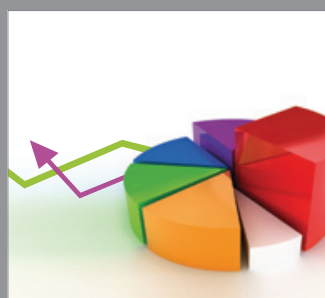

ournal of

Probability and Statistics

Promensencen
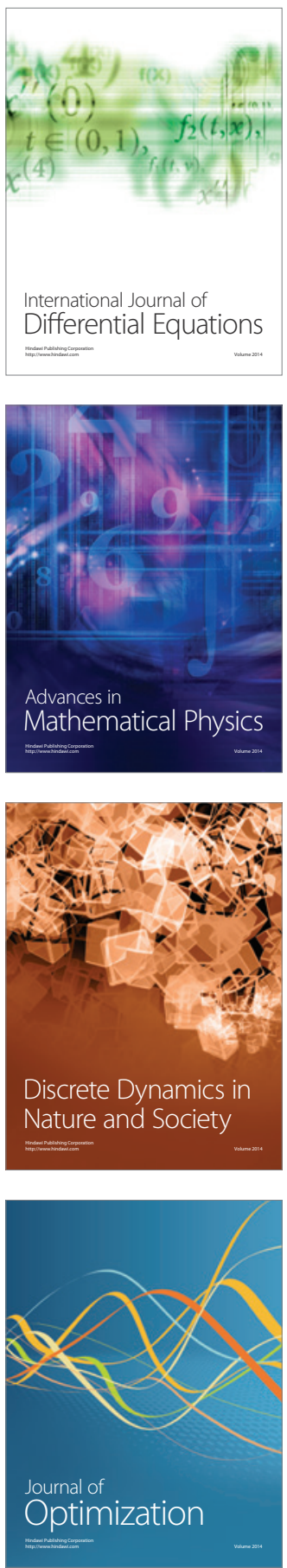